\title{
Within- and Between-Persons Effects of Self-Esteem and Affective State as Antecedents and Consequences of Dysfunctional Behaviors in the Everyday Lives of Patients With Borderline Personality Disorder
}

\author{
Philip S. Santangelo ${ }^{1,2}$, Jana Holtmann ${ }^{3}$, Georg Hosoya ${ }^{3}$, \\ Martin Bohus $^{4}$, Tobias D. Kockler ${ }^{1}$, Susanne Koudela-Hamila ${ }^{1}$, \\ Michael Eid ${ }^{3}$, and Ulrich W. Ebner-Priemer ${ }^{1}$ \\ ${ }^{1}$ Institute of Sport and Sports Science, Karlsruhe Institute of Technology; ${ }^{2}$ Department of Psychology, \\ College of Arts and Sciences, Syracuse University; ${ }^{3}$ Department of Education and Psychology, Freie \\ Universität Berlin; and ${ }^{4}$ Central Institute of Mental Health, Medical Faculty Mannheim, University \\ of Heidelberg
}

\begin{abstract}
Dysfunctional behaviors are conceptualized as maladaptive affective coping attempts in borderline personality disorder (BPD). The recent benefits-and-barriers model extended the affective function assumption by adding self-esteem as a barrier to engaging in dysfunctional behaviors. Patients with BPD $(N=119)$ carried e-diaries to report their current selfesteem, emotional valence, tense arousal, and whether they engaged in dysfunctional behaviors 12 times a day for 4 days. Dynamic structural equation modeling revealed that on the within-person level, high momentary negative affect predicted dysfunctional behaviors, and on the between-person level, low trait self-esteem predicted dysfunctional behaviors. We also found an association between engaging in dysfunctional behaviors and momentary self-esteem and trait levels of valence and tense arousal. Moreover, our results indicate a deterioration of, rather than relief from, negative affective state after dysfunctional behaviors. These findings highlight the importance of emotion-regulation skills and reestablishing a positive self-view as important treatment targets to reduce dysfunctional behaviors in BPD.
\end{abstract}

\section{Keywords}

borderline personality disorder, dysfunctional behavior, e-diary, benefits-and-barriers model, emotion-regulation model, dynamic structural equation modeling

Borderline personality disorder (BPD) is defined by a pervasive pattern of instability in four different areas: unstable affect, unstable interpersonal relationships, impulsive behaviors, and unstable sense of self (American Psychiatric Association, 2013; World Health Organization, 1992). Impulsive behaviors include nonsuicidal self-injury (i.e., the deliberate damage of bodily tissue without a lethal intent; Nock \& Favazza, 2009) as well as impulsive tendencies such as binging and vomiting, excessive alcohol and illicit drug usage, promiscuous sex, and high-risk behavior (American Psychiatric Association, 2013). These behavioral patterns are mainly seen as maladaptive coping attempts of affective dysregulation and have been termed dysfunctional behaviors (Linehan, 1993). Dysfunctional behaviors in BPD

\section{Corresponding Author:}

Philip S. Santangelo, Karlsruhe Institute of Technology, Engler-BunteRing 15, 76131 Karlsruhe, Germany

E-mail: philip.santangelo@kit.edu 
are highly prevalent (up to 90\%; e.g., Linehan, 1993; Wedig et al., 2012; Zanarini, Laudate, Frankenburg, Wedig, \& Fitzmaurice, 2013), are seen as a cause of comorbid mental disorders (e.g., substance abuse, McGlashan et al., 2000; eating disorders, Marino \& Zanarini, 2001), and have been related to suicidal behaviors (e.g., Guan, Fox, \& Prinstein, 2012; Whitlock et al., 2013).

For investigating the processes and states surrounding the occurrences of actual dysfunctional behaviors, electronic diaries (e-diaries) have become the preferred method (Santangelo, Bohus, \& Ebner-Priemer, 2014; Trull, 2018). E-diaries allow modeling of dynamic withinperson processes on the basis of multiple momentary ratings assessed in near real time (i.e., without retrospective biases) and in everyday life (i.e., with high ecological validity; Trull \& Ebner-Priemer, 2013). Empirical evidence has already shown the limited ability of retrospective measures (i.e., interviews or questionnaires) to reproduce the dynamics of experienced symptomatology (e.g., Solhan, Trull, Jahng, \& Wood, 2009) as well as the mechanisms involved in the occurrence of dysfunctional behaviors (Kockler, Santangelo, \& Ebner-Priemer, 2018; Rodríguez-Blanco, Carballo, \& Baca-García, 2018). When examining the processes involved in occurrences of dysfunctional behaviors, an utmost important concern is the sampling frequency. Impulsive behaviors, by definition, are characterized by short-term action, which make a high sampling frequency essential to disentangle antecedents and consequences of these processes over time because such short-lived dynamics may be missed by low sampling frequencies (Kockler et al., 2018). Indeed, it has been shown empirically that affective (and self-esteem) processes are quite fast in BPD (Ebner-Priemer \& Sawitzki, 2007; Santangelo et al., 2017; Santangelo, Reinhard et al., 2014), as are the processes associated with dysfunctional behaviors (e.g., in eating disorders; Kockler et al., 2018).

\section{Theoretical Models of Dysfunctional Behaviors}

The emotion-regulation model of dysfunctional behaviors (Linehan, 1993) and nonsuicidal self-injuries (e.g., Chapman, Gratz, \& Brown, 2006; McKenzie \& Gross, 2014; Nock \& Prinstein, 2004) proposes that emotion dysregulation is central to the occurrence of dysfunctional behaviors given that high levels of aversive negative emotions typically antecede nonsuicidal self-injuries, which are subsequently followed by emotional relief negatively reinforcing these behaviors (Hasking, Whitlock, Voon, \& Rose, 2017). Until now, empirical evidence has been limited, but there is an initial set of e-diary studies focused on testing the emotion-regulatory function of dysfunctional behaviors and therefore elucidating the psychological states surrounding these behaviors among patients with BPD. Selby et al. (2012) examined 25 BPD patients with comorbid bulimia nervosa using e-diaries with a combined time- and event-based sampling strategy with six random prompts daily (i.e., in approximately 2-hr intervals) for 2 weeks. Addressing the antecedents, their findings showed that negative affect increased and positive affect decreased in the 4 hr leading up to binge eating and to vomiting in the BPD patients. The results regarding the consequences revealed that levels of negative affect decreased and positive affect increased after engaging in binge eating and vomiting. Selby and Joiner (2013) used e-diaries with five random prompts per day (i.e., approximately every 2-3 hr) over 2 weeks to examine 47 behaviorally dysregulated participants, of whom 16 fulfilled the diagnosis of BPD. The findings regarding the antecedents revealed that elevated negative affect and elevated rumination prospectively predicted the occurrence of dysfunctional behaviors at the subsequent assessment (i.e., 2-3 hr later). However, for participants with high levels of BPD symptoms, negative affect at low levels of rumination was not predictive of dysfunctional behaviors, and neither were high levels of rumination at low levels of negative affect.

In addition to those two e-diary studies examining the antecedents and consequences of often reported dysfunctional behaviors in BPD in daily life, more studies examined the affective surroundings of specifically nonsuicidal self-injuries. Snir, Rafaeli, Gadassi, Berenson, and Downey (2015) analyzed 10-hr intervals preceding and following acts of nonsuicidal self-injury in 38 patients with BPD in daily life (using e-diaries with five random prompts per day over 21 days). Of those patients, 18 reported at least one nonsuicidal self-injury act during the e-diary period. Dissociation and perceived feelings of rejection and social isolation (but not negative affect) increased in the $10 \mathrm{hr}$ before acts of nonsuicidal selfinjury and faded gradually in the subsequent $10 \mathrm{hr}$ following the self-injury. Andrewes, Hulbert, Cotton, Betts, and Chanen (2017b) examined the emotional trajectories preceding and following acts of nonsuicidal self-injury in 107 patients with BPD (using e-diaries with six random prompts in approximately 2-hr intervals per day over 6 days). Analysis of 24 participants who engaged in nonsuicidal self-injuries during the assessment period revealed increased negative affect and decreased positive affect in the hours before acts of self-injuries and decreased negative affect and increased positive affect in the hours after self-injuries. Similar to Snir et al., changes in negative and positive affect occurred several hours before and after engaging in the behavior (a median of 15 and $10 \mathrm{hr}$ before self-injuries and similar 
time intervals after self-injuries). Reanalyzing the same data set, Andrewes, Hulbert, Cotton, Betts, and Chanen (2017a) found that the number of negative complex emotions experienced as well as levels of distress increased before self-injuries and decreased after them. Again, changes in negative complex emotions and distress occurred a median of 17 and $19 \mathrm{hr}$, respectively, before self-injuries and a median of 18 and $20 \mathrm{hr}$, respectively, following self-injuries. Those large time intervals between changes in affective states and selfinjuries as well as between the behaviors and the emotional relief reported in the studies above are in discordance with the reinforcement hypothesis inherent to the emotion regulation model. Moreover, they are in discord to the theoretical assumption (Linehan, 1993), diagnostic criterion (American Psychiatric Association, 2013; World Health Organization, 1992), and empirical findings (e.g., Ebner-Priemer \& Sawitzki, 2007; Santangelo et al., 2017; Santangelo, Reinhard, et al., 2014) of fast changes of affective states in BPD. Therefore, the question arises whether these studies with a rather low sampling rate actually tracked the process of interest (i.e., the affective trajectories preceding and following self-injuries and dysfunctional behaviors). A high sampling frequency is key to capture short-lived dynamics. Therefore, the e-diary study by Houben and colleagues (2017) is the most sophisticated to date. The authors examined the affective antecedents and consequences of nonsuicidal self-injuries in a small sample of 30 predominantly female inpatients with presumed BPD. Although patients resided in a psychiatric hospital, they carried an e-diary for 8 days. The e-diaries emitted a prompting signal 10 times a day, which resulted in a mean time interval of $1.33 \mathrm{hr}$. Of the 30 patients, 18 reported at least one act of nonsuicidal self-injury during the assessment period. Overall, patients reported nonsuicidal self-injuries on $5.6 \%$ of all sampled time points; there was large variability between persons ( $S D=10.19$, range $=54)$. Using multilevel models and performing lagged analyses, Houben et al. revealed that high levels of negative affect (but not low levels of positive affect) prospectively predicted a higher probability of engaging in nonsuicidal self-injuries in the next time interval. However, results regarding the consequences showed that negative affect increased after reports of self-injuries and further increased one time interval later. Therefore, nonsuicidal self-injuries were followed by a deterioration of, rather than relief from, negative affect.

Summing up the existing empirical evidence, both the numbers of participants actually engaging in dysfunctional behaviors during the e-diary assessment (range $=16-25$ patients) and the numbers of actual dysfunctional acts (0.06-0.43 acts per day per participant) were quite low in the reported studies. Moreover, most studies used a low sampling frequency e-diary protocol with five to six assessments per day and thus large time intervals of 2 to $3 \mathrm{hr}$ between assessments. Only high-frequency repeated assessments are capable of actually capturing short-lived dynamics. In addition, e-diary research so far within the context of BPD is almost solely restricted to specific forms of dysfunctional behaviors, mainly nonsuicidal self-injuries. Only one study included various forms of often reported dysfunctional behaviors in BPD (Selby \& Joiner, 2013), which is especially important because theoretical models (e.g., the emotion-regulation model) assume equal mechanisms and functions across different forms of dysfunctional behaviors. Studies revealed mixed and inconsistent findings that are likely to be related to these issues. Regarding the antecedents, most studies provided support for increased negative affect before dysfunctional behaviors (Andrewes et al., 2017a, 2017b; Houben et al., 2017; Selby et al., 2012; Selby \& Joiner, 2013) and decreased positive affect (Andrewes et al., 2017b; Selby et al., 2012); only one study did not reveal negative affect to be a significant predictor of dysfunctional behaviors (Snir et al., 2015). However, the results regarding the affective consequences of engaging in dysfunctional behaviors are less clear; some studies indicated an affect-regulating effect of dysfunctional behaviors with decreases in negative affect (Andrewes et al., 2017a, 2017b; Selby et al., 2012) and increases in positive affect (Andrewes et al., 2017b; Selby et al., 2012), whereas others revealed contradicting results with deteriorating negative affect after engaging in dysfunctional behaviors (Houben et al., 2017; Snir et al., 2015).

Because of the inability of prior models to fully explain occurrences of dysfunctional behaviors, the benefits-andbarriers model of nonsuicidal self-injury was proposed recently by Hooley and Franklin (2018). Their model assumes that nonsuicidal self-injuries constitute a transdiagnostic phenomenon that does not occur only in patients with BPD. Thus, the core tenets of the model are that (a) nonsuicidal self-injuries have affective benefits that are universal processes available to most people but that (b) the vast majority of people do not access the benefits because of physiological, psychological, and social barriers. In more detail, the model assumes different types of nonsuicidal self-injury benefits (i.e., affect regulation, gratification of self-punishment desires, social affiliation, and communication), whereas the affective benefit is central to the model. What dissuades most people from accessing these benefits and engaging in nonsuicidal self-injury is that there are several major barriers, such as a positive view of the self (i.e., regarding oneself favorably), lack of awareness about nonsuicidal self-injury, aversion to nonsuicidal self-injury 
stimuli, dislike of physical pain, and concern about social norms. According to the model, each of these barriers must be overcome for nonsuicidal self-injuries to occur. Similar to the affect regulation component being the most important benefit of nonsuicidal selfinjuries, the most important barrier to engaging in these behaviors is the positive self. A positive view of the self is linked to positive associations to the own body, which leads to protecting it (instead of doing harm to it). The model explains this specific association between the self and engaging in nonsuicidal self-injuries as follows: A negative view of oneself generates (a) the desire to stop this unpleasant affective state, (b) the belief that one deserves pain and punishment, and (c) a greater accessibility to self-injury as a coping strategy. In turn, these factors lower the physical pain barrier by engendering the motivation to select and endure painful self-injury, which leads to nonsuicidal self-injuries. Empirical evidence supports this idea because between-person findings indicate that negative associations with the self, such as self-criticism, self-blame, self-dissatisfaction, selfdisgust, and negative self-views, are linked to nonsuicidal self-injury in recent studies (see Hooley \& Franklin, 2018).

Taken together, Hooley and Franklin (2018) refined the emotion-regulation model by adding a barrier component emphasizing the importance of the view of the self. Therefore, this model conceptualizes both a between-person mechanism (i.e., a negative view of the self) and a withinperson mechanism (i.e., momentary negative affective states) as predictors of nonsuicidal self-injuries. Because the affective benefits of these behaviors are assumed to be universal processes available to most people, it is the barriers, especially the barrier of the positive association with the self, that distinguishes between those who engage in self-injuries and those who do not.

Although the clinical implications of this work have begun to be explored only recently, there are two very recent studies using e-diary methods that substantiate the model as a promising approach. Santangelo and colleagues (2017) brought new attention to the importance of self-esteem in BPD and provided empirical evidence that instability in self-esteem is heightened in BPD, that it is highly intertwined with affective instability, and that self-esteem instability does better predict general psychopathology in BPD in comparison with affective instability.

Although using a different but associated construct, Scala et al. (2018) provided first results indicating a potential association between self-injurious urges and the self. The authors examined the interplay of affective state and identity disturbances in predicting nonsuicidal self-injury urges. Similar to the previously reported studies on nonsuicidal self-injuries, the number of participants, the number of actual urges, and the assessments per day were quite limited. Thirty-six BPD patients and 18 patients with any anxiety disorder answered six random prompts per day in approximately 2-hr intervals over 21 days. Unfortunately for analytic purposes, reports of nonsuicidal self-injury urges were quite rare (84 cases in the 54 patients; i.e., $2.2 \%$ of all assessments or 1.5 urges per participant over the 21-day assessment period). However, even with these limitations, nonsuicidal self-injury urges were significantly related to identity disturbances and negative affect.

\section{Dynamic Structural Equation Modeling for Analyzing e-Diary Data}

Statistical modeling possibilities accelerate with breathtaking pace, which allow increasingly precise and complex models. This development does expose numerous statistical limitations in previously reported studies, such as not modeling symptoms over time using autoregressive relationships (Andrewes et al., 2017b; Houben et al., 2017; Selby et al., 2012; Selby \& Joiner, 2013). Recent approaches to model e-diary data focused on random-effect multilevel autoregressive and cross-lagged models (e.g., Ebner-Priemer et al., 2015; Jongerling, Laurenceau, \& Hamaker, 2015). These models are advantageous because they allow (a) separation of interindividual, time-stable, between-person differences (traits or home base) from intraindividual, within-person fluctuations around this time-stable person-specific value; (b) examination of interindividual differences in autoregressive relationships (sometimes called inertia, i.e., the speed with which a person returns to his or her trait level); (c) examination of interindividual differences in cross-lagged/predictive relationships (random slopes); and (d) examination of interindividual differences in the reactivity to unobserved influences (random innovation variances). However, random-effect multilevel autoregressive and cross-lagged models still have several limitations. Most importantly, (a) because they are not able to model latent variables, unexplained systematic occasion-specific variability is inseparably confounded with unsystematic measurement error (i.e., true instability cannot be separated from measurement error, thereby increasing instability estimates), and (b) observations are assumed to be equidistant, which is rarely the case in e-diary studies.

New developments in structural equation modeling (SEM) now allow for the estimation of multilevel, randomeffect SEM with lagged relationships of latent factors on the within-person level, thereby taking measurement error into account. Dynamic SEM (Asparouhov, Hamaker, \& Muthén, 2018) combines time-series modeling with multilevel SEM, which allows modeling dynamic patterns of latent variables while taking nonequidistant observations into account. This ability is especially important because 
the strength of a lagged or cross-lagged effect depends on the time interval between measurement occasions. In the collection of e-diary data, unequal time intervals between measurements are frequent because of missed responses and the inevitable periods of missing measurements during the night. In dynamic SEM, a continuous time dimension is approximated, missing values are augmented, and models are estimated using Markov chain Monte Carlo algorithms and Bayesian estimation techniques (Asparouhov et al., 2018).

\section{Current Investigation}

We used state-of-the-art methodology (e-diaries with a high sampling frequency) and statistics (dynamic SEM taking into account person-specific cross-lagged effects including random innovation variances and considering unequal time intervals between measurements) to investigate the effects of affect and self-esteem as they relate to dysfunctional behaviors in patients with BPD. We derived four hypotheses from the benefits-andbarriers model, whereby we broaden the scope of the model and test its assumptions examining dysfunctional behaviors in general (and not only nonsuicidal selfinjury). We hypothesized that (a) a negative affective state is associated with a higher probability of engaging in dysfunctional behaviors on the following assessment in patients with BPD (i.e., we assumed negative affective state to be the main within-subject antecedent of dysfunctional behaviors). Because the benefits-andbarriers model further postulates that only subjects with low self-esteem engage in dysfunctional behaviors, we hypothesized that (b) predominantly low trait selfesteem across the assessment period is associated with a higher probability of engaging in dysfunctional behaviors on the between-person level. Or said in simple terms, we assumed that patients high on self-esteem do not engage in dysfunctional behaviors. Moreover, we aimed to address (c) the joint predictive effect of affect and self-esteem on subsequent occurrences of dysfunctional behaviors. To address the emotion-regulatory effect of those behaviors (i.e., the consequences), we hypothesized that (d) affective state improves after reports of dysfunctional behaviors (i.e., we assumed a more positive affective state directly after reports of dysfunctional behaviors on the within-person level).

\section{Method}

\section{Sample}

The sample consisted of 119 female patients with BPD ages 18 to 45 years (mean age $=27.9$ years, $S D=7.57$ ). Table S1 in the Supplemental Material available online provides further detail on the demographics of the participants in this sample. All participants met the diagnostic criteria from the fourth edition of the Diagnostic and Statistical Manual of Mental Disorders (DSM-IV; American Psychiatric Association, 1994) for BPD. Patients with histories of schizophrenia, bipolar disorder, or current substance abuse were excluded. Female patients with BPD were recruited from the waiting list for a residential treatment program at the Central Institute of Mental Health Mannheim, Germany. The study protocol was approved by the Ethical Committee of the Medical Faculty, Heidelberg University, Germany, and all participants provided written informed consent before inclusion in the study.

All patients were diagnosed using the German versions of the Structured Clinical Interview for $D S M-I V$ Axis I disorders (SCID-I; Wittchen, Wunderlich, \& Gruschwitz, 1997) and Axis II disorders (SCID-II; Fydrich et al., 1997). The $D S M-I V$ diagnostic criteria for BPD correspond to the diagnostic criteria as stated in the fifth edition of the Diagnostic and Statistical Manual of Mental Disorders (DSM-5; American Psychiatric Association, 2013). Both the SCID-I and the SCID-II are well-validated diagnostic instruments with very good psychometric properties (i.e., SCID-I mean $\kappa>.71$; SCID-II mean $\kappa>$.84; Lobbestael, Leurgans, \& Arntz, 2011). Experienced and well-trained postgraduate psychologists administered the diagnostic instruments. As Table S1 shows, comorbidities were common in the patients with BPD. The most frequent comorbid Axis I diagnoses included mood disorders $(n=88,73.9 \%)$ and anxiety disorders $(n=76,56.3 \%)$.

\section{E-diary assessment and measures}

Data on momentary self-esteem, affective state, and dysfunctional behaviors were collected during participants' daily lives. After completing the diagnostic assessments, participants received an e-diary. Slightly more than half of the participants (54.6\%) received a palmtop computer (Tungsten-E, Palm Inc., USA) programmed with the IzyBuilder software (IzyData Ltd., Switzerland), whereas the rest of the sample (45.4\%) received a study smartphone programmed with the movisensXS app (movisens $\mathrm{GmbH}$, Karlsruhe, Germany). We checked for basic differences between the assessment devices and found no differences (cf. Santangelo et al., 2018). All participants were thoroughly instructed and trained regarding the use of the e-diary. Participants carried the e-diary on 4 consecutive days. The e-diary emitted a prompting signal according to a pseudorandomized time-sampling schedule in hourly intervals $(60 \mathrm{~min} \pm 10 \mathrm{~min})$ from 10 a.m. to 10 p.m. Participants were prompted 12 times a day, which resulted in a total of 48 prompts per participant over the 4-day assessment period. Each response was automatically time-stamped by the e-diary. After 
completing 4 assessment days, participants returned the e-diaries and were debriefed and financially compensated (€40-€50) according to the number of completed data entries.

At each prompt, participants rated their current affect, self-esteem, and occurrences of dysfunctional behaviors since the last prompt. To assess participants' momentary affective states, we used a specifically designed and validated measure for repeated assessments of momentary affective states in e-diary studies (Wilhelm \& Schoebi, 2007). Momentary affective state was conceptualized as varying along two dimensions, and participants rated two bipolar items for each valence (range $=$ unpleasant-pleasant) and tense arousal $($ range $=$ restless $/$ under tension-calm $/$ relaxed $)$. Item wordings of the valence scale were the German equivalent of "At this moment I feel: unwell-well" and "content-discontent" (reverse-coded) and of the tense arousal scale, "At this moment I feel: agitated-calm" and "relaxed-tense" (reverse-coded). Patients with a palmtop computer rated the four bipolar items regarding their momentary affective state on a 7 -point rating scale ranging from 0 to 6 , whereas those with a study smartphone rated each item on a visual analogue scale ranging from 0 to 100 . To yield comparable values, ratings of the visual analogue scale (0-100) were converted into the 7-point rating scale (0-6) for the four items.

To assess participants' current view of themselves (i.e., their state self-esteem), we used a four-item short form of the Rosenberg Self-Esteem Scale (Rosenberg, 1965). Items 1, 2, 9, and 10 were adapted to assess patients' current status. The wording of the items used was the German equivalent of "At the moment: ...I am satisfied with myself; ...I think I am no good at all; ...I am inclined to feel that I am a failure; and ...I take a positive attitude toward myself." (The second and third statements were reverse-coded.)

The original 4-point rating scale was expanded to increase the potential variability in the ratings (see Borton, Crimmins, Ashby, \& Ruddiman, 2012). More specifically, patients with a palmtop computer rated the four items on a 10-point rating scale ranging from 0 to 9, whereas those with a study smartphone rated each item on a visual analogue scale ranging from 0 to 100 . To yield comparable values, ratings of the visual analogue scale (0-100) were converted into the 10-point rating scale (0-9). The items to assess participants' momentary affective states and self-esteem have been successfully used in prior studies and shown high reliability of within-person changes over time (McDonald's $\omega$ coefficients: valence $\omega=0.75-0.79$, tense arousal $\omega=0.7-0.75$, self-esteem $\omega=0.83$; Santangelo et al., 2017; Santangelo et al., 2018).
To assess occurrences of dysfunctional behavior, participants were asked to report instances of dysfunctional behavior since the last prompt. We used a list of often reported dysfunctional behaviors that we retrieved from the DSM criteria for BPD (American Psychiatric Association, 2013). The wording of this item was, "Did you engage in dysfunctional behavior since the last prompt?" In case this item was answered with yes, participants were presented the following list, from which they could select one or more dysfunctional behaviors: "Which of the following dysfunctional behaviors have you engaged in? cutting/scratching/burning; banging head; binging/vomiting; alcohol, drugs; sexual impulsivity; high-risk behavior; other form of dysfunctional behavior."

For the variables included in the analyses, possible values ranged from 0 to 6 for valence and tense arousal and from 0 to 9 for self-esteem. The variable dysfunctional behavior was coded as a dummy variable: 1 was used for reports of dysfunctional behaviors and 0 was used when no dysfunctional behaviors were reported.

\section{Data preprocessing and statistical modeling}

All items were recoded such that high values on items of valence indicate high pleasantness, high values on items of tense arousal indicate high calmness (i.e., low tension), and high values on items of self-esteem indicate high self-esteem. For each of the three constructs, item parcels were built such that a parcel is the mean value across all available items for the respective construct.

The dynamic interplay of dysfunctional behavior, affect, and self-esteem was analyzed using dynamic SEM. These dynamic SEMs include latent autocorrelations and cross-lagged effects of all variables at the within-person level, allow for person-specific random innovation variances (variances of the time-varying within-person variables that cannot be explained by the dynamic process, i.e., preceding time points through autoregressive and cross-lagged effects), and take varying time intervals between measurements into account. The model employed is an extension of the model introduced by and explained in detail in Schuurman and Hamaker (2019). In the following, we focus on the most important aspects of the model definition (for further details, see the Model Definitions section and the Mplus Priors for Dynamic SEM section in the Supplemental Material).

In a first step, dysfunctional behavior was combined with either tense arousal, valence, or self-esteem into one model (Models 1-3). In a second step, dysfunctional behavior was combined with one affect dimension (tense 
arousal or valence) as well as self-esteem in one dynamic SEM model (Models 4 and 5). This two-step procedure was adopted to reduce model complexity in Models 1 to 3 and to ensure that the observed patterns of effects replicate across the extended models and their reduced, comparatively less complex counterparts. In the following, we describe the model combining dysfunctional behavior with tense arousal and self-esteem (Model 5). Model 4 is analogous and replaces tense arousal by valence.

Latent decompositions. According to a multilevel data structure, each observed continuous variable is decomposed into a latent person-specific mean, a time-specific within-person deviation from this person-specific mean, and a measurement error term. The idea of this approach is quite similar to centering in random regression modeling while taking into account measurement error. For instance, let $Y_{\text {TAit }}$ be the observed variable for tense arousal and $Y_{S i t}$ the observed variable of self-esteem for individual $i$ at time $t$, which are decomposed in the following way:

$$
\begin{gathered}
Y_{T A i t}=\mu_{T A i}+T A_{i t}+\varepsilon_{T A i t} \\
Y_{S i t}=\mu_{S i}+S_{i t}+\varepsilon_{S i t},
\end{gathered}
$$

in which $\mu_{T A i}$ and $\mu_{S i}$ are the latent person-specific means (stable traits) of tense arousal and self-esteem (i.e., the expected values of $Y_{\text {TAit }}$ and $Y_{\text {Sit }}$ for person $i$ over all time points), and $T A_{i t}$ and $S_{i t}$ are the timespecific deviations of $Y_{\text {TAit }}$ and $Y_{S i t}$ from their respective person-specific stable means. $\varepsilon_{T A i t}$ and $\varepsilon_{S i t}$ denote measurement error terms, which are assumed to be multivariate normally distributed with person-specific variances and covariances ${ }^{1}$ (Schuurman \& Hamaker, 2019):

$$
\left(\begin{array}{c}
\varepsilon_{\text {TAit }} \\
\varepsilon_{\text {Sit }}
\end{array}\right) \sim \operatorname{MVN}\left(\left[\begin{array}{l}
0 \\
0
\end{array}\right],\left[\begin{array}{cc}
\sigma_{\varepsilon T A i}^{2} & \sigma_{\varepsilon T A S i}^{2} \\
\sigma_{\varepsilon T A S i}^{2} & \sigma_{\varepsilon S i}^{2}
\end{array}\right]\right)
$$

for all $t$ (i.e., $\varepsilon_{\text {TAit }}$ and $\varepsilon_{\text {Sit }}$ are identically and independently distributed across time points). Whereas the time-specific within-person deviations (e.g., $T A_{i t}$ ) are measured on the within-person level to study intraindividual temporal dynamics, the person-specific means (trait variables; e.g., $\mu_{T A i}$ ) are modeled on the betweenperson level to study interindividual differences.

Analogously, the dichotomous variable dysfunctional behavior is decomposed into a person-specific expectation, $\mu_{D B i}$, across time and a time-specific deviation from this person-specific expectation, $D B_{i t}$, within a probit regression. That is, the probability that person $i$ at time $t$ engages in dysfunctional behavior is connected to a person-specific latent trait and a person-and-time-specific latent deviation from this person-specific trait. Assuming a latent continuous response variable $Y_{D B i t}^{*}$ underlying the dichotomous variable of reporting or not reporting dysfunctional behavior at time point $t$ for individual $i$,

$$
\begin{gathered}
P\left(Y_{D B i t}=1\right)=P\left(Y_{D B i t}^{*}>\tau\right) \\
Y_{D B i t}^{*}=\mu_{D B i}+D B_{i t},
\end{gathered}
$$

dysfunctional behavior is presumed if the latent response variable surpasses a certain threshold $\tau$ - that is, $Y_{D B i t}=1$ if $Y_{D B i t}^{*}>\tau$, and $Y_{D B i t}=0$ if $Y_{D B i t}^{*} \leq \tau$. Note that Equation 5 does not include an error term because measurement error and dynamic error are not separated for the dichotomous variable $Y_{D B i t}$, and the respective error term is included in the within-person dynamics of dysfunctional behavior (see Equation 8).

Within-person dynamics. On the within-person level, the models include autoregressive effects of order 1, $A R(1)$, as well as fully crossed-lagged effects on all included variables (with the exception of the error variables). That is, all within-level latent variables $\left(T A_{i}, S_{i t}, D B_{i t}\right)$ are used as antecedents to predict current states of affect, self-esteem, and dysfunctional behavior. For instance, the time-specific, within-part of tense arousal at time point $t$ is regressed on the time-specific latent tense arousal, self-esteem, as well as dysfunctional behavior of the previous measurement time point. Because we included the autoregressive and cross-lagged effects, the dependency of within-person observations over time is taken into account in the model estimation. All autoregressive and cross-lagged effects are random effects (i.e., person-specific). The within-person dynamic process can be expressed as

$$
\begin{gathered}
T A_{i t}=\varphi_{i T A} T A_{i(t-1)}+\varphi_{i T A O n S} S_{i(t-1)}+\varphi_{i T A O N D B} D B_{i t}+\zeta_{T A i t} \\
S_{i t}=\varphi_{i S O n T A} T A_{i(t-1)}+\varphi_{i S} S_{i(t-1)}+\varphi_{i S O n D B} D B_{i t}+\zeta_{S i t} \\
D B_{i t}=\varphi_{i D B o n T A} T A_{i(t-1)}+\varphi_{i D B o n S} S_{i(t-1)}+\varphi_{i D B} D B_{i(t-1)}+\varepsilon_{D B i t} .
\end{gathered}
$$

Note that the time index for the cross-lagged effects of dysfunctional behavior on tense arousal and self-esteem is $t$ (and not $t-1$ ) because the occurrence of dysfunctional behavior within the time period preceding affect and selfesteem is measured retrospectively (i.e., at measurement 
time point $t$, participants indicated how they felt at that exact moment and whether dysfunctional behavior had occurred since the last report). Hence, dysfunctional behavior is located in between two affect or self-esteem measures on the timeline. Because we included the autoregressive and cross-lagged parameters as random effects, the dynamic temporal associations between tense arousal, self-esteem, and the probability to engage in dysfunctional behavior may vary across persons. $\zeta_{A i t}$ and $\zeta_{S i t}$ are random residual variables, also called innovations, disturbances, or dynamic errors, which capture the withinperson variance that cannot be explained by carryover effects (i.e., by the autoregressive and cross-lagged effects of temporally preceding variables). The innovations thereby capture the effect of unobserved sources on a variable's development over time and may affect the system across several occasions. They are assumed to be multivariate normally distributed with

$$
\left(\begin{array}{c}
\zeta_{\text {TAit }} \\
\zeta_{\text {Sit }}
\end{array}\right) \sim M V N\left(\left[\begin{array}{l}
0 \\
0
\end{array}\right],\left[\begin{array}{cc}
\sigma_{\zeta T A i}^{2} & \sigma_{\zeta T A S i}^{2} \\
\sigma_{\zeta T A S i}^{2} & \sigma_{\zeta S i}^{2}
\end{array}\right]\right)
$$

for all $t$ (identically and independently distributed across time). As indicated by the index $i$, the variances and covariances of $\zeta_{\text {TAit }}$ and $\zeta_{\text {Sit }}$ can vary across persons. ${ }^{2}$ Because we included these person-specific variances, individual differences in the variability of exposure and reactivity to unobserved influences are modeled (Jongerling et al., 2015). Note that for the latent variable underlying dysfunctional behavior, innovation and measurement error variance are not separated, and all unexplained variance is captured by the error term $\varepsilon_{D B i t}$ in Equation (8), with $\varepsilon_{D B i t} \sim N(0,1){ }^{3}$

Figure 1 depicts the within-level of the dynamic SEM for the combination of dysfunctional behavior and one affect variable. Figure 2 depicts the within-level model combining dysfunctional behavior with one affect variable and self-esteem. Note that the between-level covariance structure is not depicted in the figures. Models 1,2 , and 3 are analogous to the model depicted in Figure 1 and replace the affect variable by tense arousal (Model 1), valence (Model 2), or self-esteem (Model 3). Models 4 and 5 are analogous to the model depicted in Figure 2 and replace the affect variable by either tense arousal (Model 4) or valence (Model 5).

Between-person covariance structure. All personspecific autoregressive and cross-lagged parameters $\varphi_{i}$ (referring to $\varphi_{i T A} \varphi_{i S}, \varphi_{i D B}, \varphi_{i T A O n D B}, \varphi_{i S O n D B}, \varphi_{i T A o n S}, \varphi_{i S o n T A}$, $\left.\varphi_{i D B N T A}, \varphi_{i D B o n S}\right)$ are estimated with a mean and a variance on the between-person level:

$$
\varphi_{i}=\gamma_{\varphi}+u_{\varphi i}
$$

in which $\gamma_{\varphi}$ is the mean slope over all persons (fixed effect) and $u_{\varphi i}$ is the deviation of the person-specific slope $\varphi_{i}$ from the mean slope (random effect; Hamaker, Asparouhov, Brose, Schmiedek, \& Muthén, 2018). All person-specific autoregressive and cross-lagged effects are assumed to be multivariate normally distributed along with the person-specific trait variables $\mu_{T A i}$ and $\mu_{S i}$. That is, stable trait characteristics can correlate with individual inertia and cross-lagged parameters in modeling interindividual differences.

The latent trait component of dysfunctional behavior is regressed on the latent trait variables of self-esteem and tense arousal on the between-person level-that is,

$$
\mu_{D B i}=\beta_{T A} \mu_{T A i}+\beta_{S} \mu_{S i}+\delta_{D B i}
$$

in which $\beta_{T A}$ and $\beta_{S}$ are regression coefficients representing the effect of trait tense arousal and self-esteem on the probability to show dysfunctional behavior, and $\delta_{D B i}$ is a regression residual with $\operatorname{Var}\left(\delta_{D B i}\right)=\theta$. The person-specific trait levels of tense arousal and selfesteem thereby serve to explain the general tendency of a person to engage in dysfunctional behavior (stable trait component).

\section{Model estimation}

All dynamic SEM models were estimated using the new dynamic SEM feature (Asparouhov et al., 2018) and Bayesian estimation techniques in Mplus (Version 8.3; Muthén \& Muthén, 2017). Because the prompting signal for data collection was emitted on average every 60 min, the tinterval option of Mplus was set to $60 \mathrm{~min}$; that is, the time grid for estimation is based on intervals of $1 \mathrm{hr}$. Therefore, missing observations are inserted in 1-hr intervals for the time periods between days (i.e., from an evening until the morning of the following day) as well as for missing responses within a day. That is, the model corrects for nonequidistant time intervals between measurements. For a detailed description of the algorithm implemented in Mplus for approximating a continuous time model with a discrete time dynamic SEM model by insertion of missing data and rescaling of the time variable, see Asparouhov et al. (2018).

All Bayesian analyses were conducted using diffuse priors (i.e., the Mplus default priors; for details, see Mplus Priors for Dynamic SEM in the Supplemental Material). All dynamic SEMs were estimated using two Monte Carlo chains with at least 200,000 iterations and using only every 20th iteration for parameter inference in the posterior distribution. The first half of each chain was discarded as burn-in, which means that results are based on at least 10,000 posterior draws. Convergence was assumed if the potential scale-reduction factor 


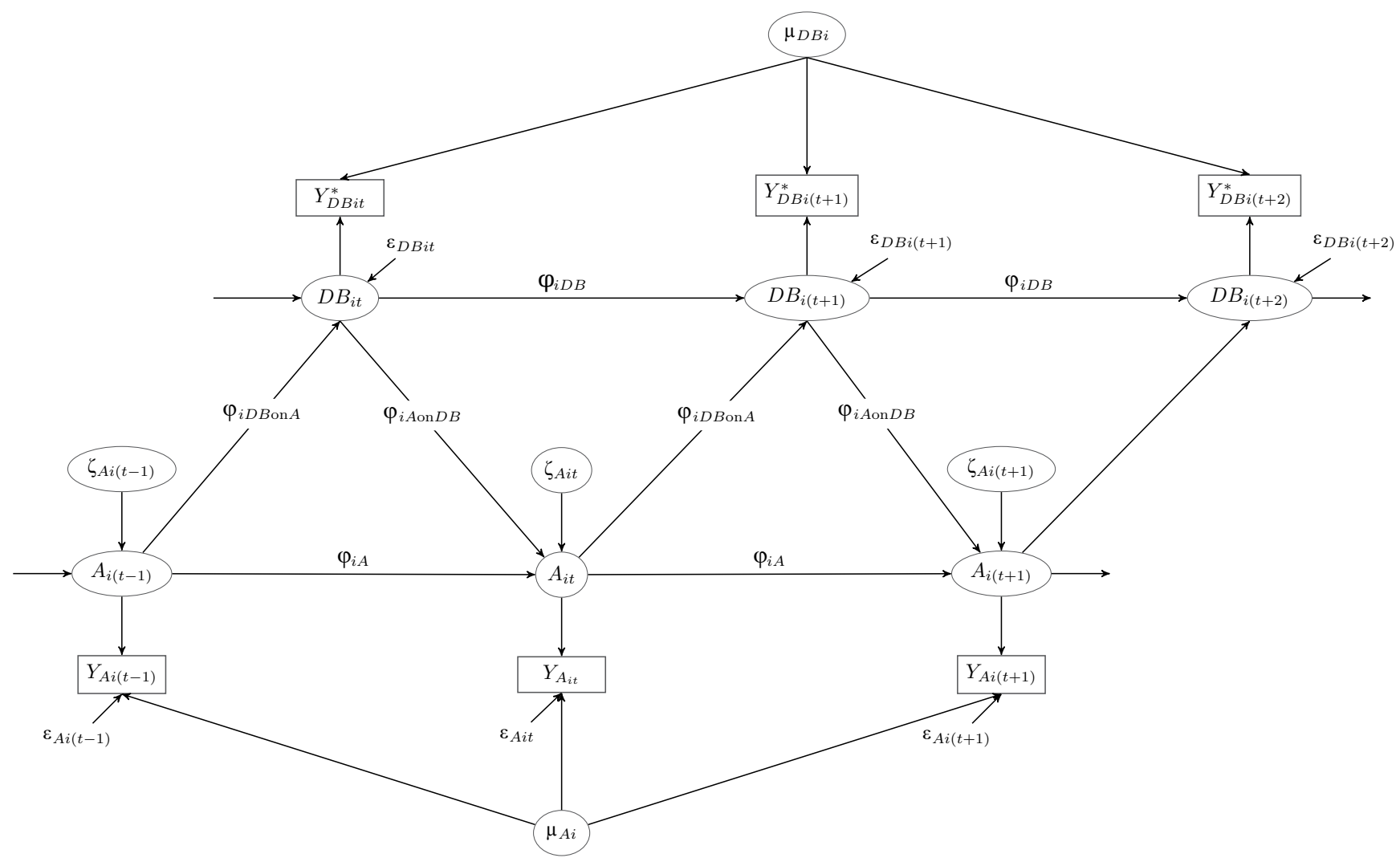

Fig. 1. Dynamic structural equation modeling of dysfunctional behavior (DB) and affect (A). Because dysfunctional behavior is a dichotomous observed variable, all regressions including this variable are probit regressions and depicted for the underlying latent continuous response variable $Y_{D B i t}^{*}$. The observed variable $Y_{A i t}$ and the latent response variable of the probit regression $Y_{D B i t}^{*}$ are decomposed into their respective between- $\left(\mu_{A i}\right.$ and $\left.\mu_{D B i}\right)$ and within-person $\left(A_{i t}\right.$ and $\left.D B_{i t}\right)$ parts as well as measurement error variables $\left(\varepsilon_{A i t}\right.$ and $\varepsilon_{D B i t}$; the measurement error variance for affect is person-specific). The model includes random autoregressive effects $\left(\varphi_{i A}\right.$ and $\left.\varphi_{i D B}\right)$ as well as cross-lagged effects ( $\varphi_{i A o n D B}$ and $\varphi_{i D B \text { onA }}$ ) of order 1 on the within-person level; that is, $A_{i t}$ at time point $t$ is regressed on $A_{i(t-1)}$ as well as $D B_{i t}$, and $D B_{i t}$ at time point $t$ is regressed on $D B_{i(t-1)}$ as well as $A_{i(t-1)}$. Note that $D B_{i t}$ temporally precedes $A_{i t}$, although measured at the same measurement occasion, because dysfunctional behavior is assessed as "since the last measurement" and affect is assessed as "in this moment." The autoregressive and cross-lagged regression slopes are modeled as random effects; that is, they are person-specific. In addition, the variance of the innovation term of affect $\left(\zeta_{A i t}\right)$ is modeled as a random effect (i.e., the amount of variance in affect that is not explained by the autoregressive and cross-lagged effects is person-specific). Note that regressions and covariance structure of the between-level of the model are not depicted in the figure. On the between-person level, the probability of showing DB $\left(\mu_{D B}\right)$ is regressed on the person-specific $\left(\right.$ stable) trait of affect $\left(\mu_{A}\right)$. All other random effects (except for the measurement error variances) are allowed to correlate on the between-person level.

(Asparouhov \& Muthén, 2010) fell below the cutoff of 1.01 for all parameters and was checked by visual inspection of Bayesian posterior parameter trace plots for each parameter. The median of the posterior distribution was used as a point estimate, and posterior quantiles were used to provide a $95 \%$ credibility interval for the parameter estimates. To compare the strength of the cross-lagged associations, standardized parameter estimates were used. According to the recommendation by Schuurman, Ferrer, Boer-Sonnenschein, and Hamaker (2016), we focused on within-person standardized effects for the comparison of the strengths of within-person cross-lagged effects. That is, standardized coefficients for the within-person, individual-specific effects are standardized per person and then averaged across persons (Schuurman et al., 2016).

\section{Results}

\section{Preliminary analyses}

Overall compliance with the hourly assessments was very good (84.86\% completed prompts). Participants provided on average, 40.73 self-reports and in total, 4,847 reports (see Table S1 in the Supplemental Material). During the 4 assessment days, patients with BPD reported on average, 7.01 instances of dysfunctional behaviors (median $=4.00$ ) and in total, 834 reports. Thus, on 834 out of 4,847 assessment occasions (i.e., $16.68 \%$ ), patients with BPD reported engaging in a total of 1,227 dysfunctional behaviors (because reports of different dysfunctional behaviors on one assessment occasion were possible). Table S1 shows the frequencies of the different dysfunctional behaviors that were 


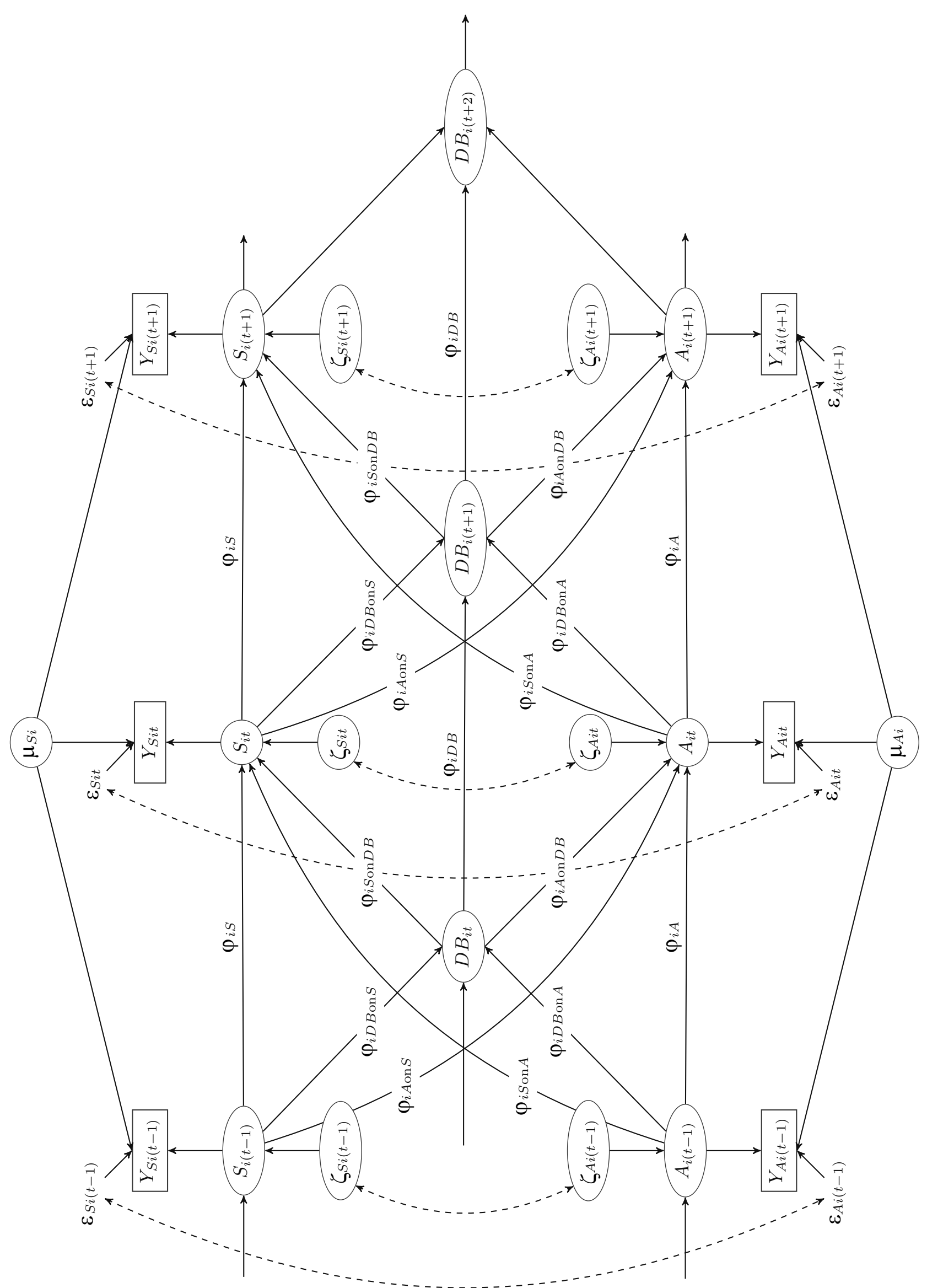

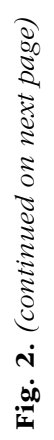


Fig. 2. Dynamic structural equation modeling of dysfunctional behavior (DB), affect (A), and self-esteem (S). Because dysfunctional behavior is a dichotomous observed variable, all regressions including this variable are probit regressions. The observed variables $Y_{A i t}$ and $Y_{S i t}$ and the latent response variable of the probit regression $Y_{D B i t}^{*}$ are decomposed into their respective between- $\left(\mu_{A i}\right.$, $\mu_{S i}$ and $\left.\mu_{D B i}\right)$ and within-person $\left(A_{i t}, S_{i t}\right.$ and $\left.D B_{i t}\right)$ parts as well as measurement error variables $\left(\varepsilon_{A i t}, \varepsilon_{D B i t}\right.$ and $\varepsilon_{S i t}$; measurement error variances for affect and self-esteem are person-specific). Note that for dysfunctional behavior, the latent decomposition in a between- and a within-person part as well as the measurent error variable are not depicted because of space restrictions. Instead, only the latent within-person variable of dysfunctional behavior $\left(D B_{i t}\right)$ is shown in the figure. The model includes random autoregressive effects $\left(\varphi_{i A}, \varphi_{i S}\right.$, and $\left.\varphi_{i D B}\right)$ as well as cross-lagged effects $\left(\varphi_{i A o n D B}\right.$, $\left.\varphi_{i S O n D B}, \varphi_{i D B \text { onA }}, \varphi_{i D B o n S}, \varphi_{i A \text { ons }}, \varphi_{i S o n A}\right)$ of order 1 on the within-person level; that is, the within-level parts of all variables at time point $t$ are regressed on the within-level parts of all other variables of the preceding time point. Note that $D B_{i t}$ temporally precedes $A_{i t}$ and $S_{i t}$, although measured at the same measurement occasion, because dysfunctional behavior is assessed as "since the last measurement" and affect and self-esteem are assessed as "in this moment." The autoregressive and cross-lagged regression slopes are modeled as random effects; that is, they are person-specific. In addition, the variances of the innovation terms of affect and self-esteem $\left(\zeta_{A i t}\right.$ and $\left.\zeta_{S i t}\right)$ are modeled as random effects (i.e., the amount of variance in affect and self-esteem that is not explained by the autoregressive and cross-lagged effects is personspecific). Measurement error terms as well as innovations (i.e., the unexplained parts of affect and self-esteem at time point $t$ ) are allowed to correlate across constructs (affect and self-esteem) within one time point (dotted lines). Note that regressions and covariance structure of the between-level of the model are not depicted in the figure. On the between-person level, the probability of showing DB ( $\mu_{D B}$ ) is regressed on the person-specific (stable) trait of affect and self-esteem $\left(\mu_{A i}\right.$ and $\mu_{S i}$ ). All other random effects (except for the measurement error and innovation variances and covariances) are allowed to correlate on the between-person level.

reported. Of the 119 patients with $\mathrm{BPD}, 19$ participants (i.e., 16.00\%) did not report engaging in dysfunctional behaviors during the assessment days. All dynamic SEMs converged well, as indicated by potential scalereduction-factor values and visual inspection of trace plots.

\section{Momentary states predicting upcoming dysfunctional behavior (Hypothesis 1; Models 1-3; Table 1)}

As hypothesized, momentary valence and tense arousal predicted upcoming dysfunctional behaviors (Models 1 and 2; Table 1). The results exhibited significant and negative probit regression coefficients $\left(\varphi_{D B D_{C} C}\right)$; that is, the probability to report dysfunctional behaviors increases with lower values of valence and tense arousal in the hour preceding the behaviors (please note that all scales were coded such that high values correspond to positive states, i.e., positive valence, high calmness/ low tension, and high self-esteem). In addition, we investigated the influence of momentary self-esteem on dysfunctional behaviors (Model 3; Table 1). Again, the results revealed a negative probit regression coefficient showing that low momentary self-esteem predicted upcoming dysfunctional behaviors. A comparison of all three within-person standardized probit regression coefficients revealed that the impact on occurrences of dysfunctional behaviors is highest for tense arousal, followed by self-esteem, and is smallest for valence in the time period directly preceding the behavior (withinperson standardized $\varphi_{\text {DBonC }}$ of $-0.165,-0.142$, and -0.121 , respectively; see Table 1). Accordingly, the model with tense arousal explained the largest amount of variance in dysfunctional behavior on the withinperson level, as $R^{2}$ values indicate (Table 1 ). That is, when controlling for autoregressive dependencies, tense arousal in the assessment directly preceding the behavior is most predictive of reporting dysfunctional behavior $\left(R^{2}=25.1 \%\right)$ compared with valence $\left(R^{2}=\right.$ $21.8 \%)$ or self-esteem $\left(R^{2}=22.9 \%\right)$.

\section{Trait self-esteem predicting dysfunctional behavior (Hypothesis 2; Models 1-3; Table 1)}

As hypothesized, between-person-level self-esteem showed a significant effect on dysfunctional behavior in the expected direction (between-person standardized $\beta$ of -0.511 ; Table 1 ). That is, the higher a person's trait level on self-esteem, the less likely it is that this person shows dysfunctional behavior in general. In addition, we found that trait levels of tense arousal and valence showed significant effects on dysfunctional behavior in the expectable direction (between-person standardized $\beta$ of -0.445 and -0.533$)$. That is, the higher a person's trait level of valence and tense arousal, the less likely it is that this person shows dysfunctional behavior in general. It is apparent, on the basis of comparisons of the standardized coefficients of $\beta$, that trait self-esteem and valence have a higher impact on the general disposition to show dysfunctional behavior compared with tense arousal (see Table 1). Accordingly, self-esteem and valence explained more variance in dysfunctional behavior (26.1\% and $28.5 \%$, respectively) compared with tense arousal (19.8\%).

\section{Combining momentary affective state and trait self-esteem to predict dysfunctional behavior (Hypotbesis 3; Models 4 and 5; Table 2)}

Valence and self-esteem. In the model combining valence and self-esteem, within-person standardized cross-lagged effects indicated that a momentary increase in self-esteem within the preceding hour reduced the probability to subsequently engage in dysfunctional behavior (within-person standardized $\varphi_{D B o n S}=-0.180$ ), whereas momentary valence 
Table 1. Results of the Dynamic SEMs Explaining Dysfunctional Behavior (DB) by One of the Predictor Variables Valence, Tense Arousal, or Self-Esteem

\begin{tabular}{|c|c|c|c|c|c|c|}
\hline \multirow[b]{2}{*}{ Parameter } & \multicolumn{2}{|c|}{ Model 1: Valence } & \multicolumn{2}{|c|}{ Model 2: Tense arousal } & \multicolumn{2}{|c|}{ Model 3: Self-esteem } \\
\hline & Estimate & $95 \% \mathrm{CI}$ & Estimate & $95 \% \mathrm{CI}$ & Estimate & $95 \% \mathrm{CI}$ \\
\hline \multicolumn{7}{|c|}{ Unstandardized parameter estimates } \\
\hline$\beta$ & -0.476 & {$[-0.690,-0.278]$} & -0.390 & {$[-0.607,-0.198]$} & -0.237 & {$[-0.340,-0.140]$} \\
\hline$E\left(\mu_{C}\right)$ & 2.719 & {$[2.531,2.908]$} & 2.789 & {$[2.620,2.958]$} & 4.111 & {$[3.788,4.433]$} \\
\hline $\operatorname{Var}\left(\mu_{C}\right)$ & 0.872 & {$[0.648,1.178]$} & 0.719 & {$[0.536,0.974]$} & 2.962 & {$[2.283,3.926]$} \\
\hline$\tau$ & 0.093 & {$[-0.486,0.652]$} & 0.332 & {$[-0.268,0.882]$} & 0.427 & {$[0.000,0.844]$} \\
\hline$\theta$ & 0.499 & {$[0.304,0.813]$} & 0.444 & {$[0.279,0.731]$} & 0.470 & {$[0.284,0.759]$} \\
\hline \multicolumn{7}{|c|}{ Fixed effects } \\
\hline$\gamma_{\varphi C}$ & 0.549 & {$[0.476,0.618]$} & 0.545 & {$[0.465,0.622]$} & 0.476 & {$[0.392,0.555]$} \\
\hline$\gamma_{\varphi D B}$ & 0.203 & {$[0.094,0.311]$} & 0.208 & {$[0.098,0.316]$} & 0.169 & {$[0.051,0.283]$} \\
\hline$\gamma_{\varphi D B o n C}$ & -0.152 & {$[-0.259,-0.056]$} & -0.231 & {$[-0.365,-0.114]$} & -0.117 & {$[-0.232,-0.014]$} \\
\hline$\gamma_{\varphi C o n D B}$ & -0.238 & {$[-0.328,-0.153]$} & -0.198 & {$[-0.285,-0.118]$} & -0.434 & {$[-0.553,-0.319]$} \\
\hline$\gamma_{\sigma \zeta C}$ & -0.657 & {$[-1.027,-0.337]$} & -0.813 & {$[-1.204,-0.472]$} & -0.656 & {$[-1.160,-0.234]$} \\
\hline$\gamma_{\sigma \varepsilon C}$ & -1.174 & {$[-1.655,-0.785]$} & -0.854 & {$[-1.262,-0.506]$} & -0.558 & {$[-1.025,-0.178]$} \\
\hline \multicolumn{7}{|c|}{ Random-effects variances } \\
\hline$\varphi_{C}$ & 0.050 & {$[0.034,0.074]$} & 0.049 & {$[0.033,0.074]$} & 0.070 & {$[0.046,0.104]$} \\
\hline$\varphi_{D B}$ & 0.111 & {$[0.069,0.173]$} & 0.131 & {$[0.085,0.196]$} & 0.131 & {$[0.083,0.201]$} \\
\hline$\varphi_{\text {DBonC }}$ & 0.064 & {$[0.037,0.115]$} & 0.084 & {$[0.047,0.160]$} & 0.060 & {$[0.036,0.104]$} \\
\hline$\varphi_{C o n D B}$ & 0.096 & {$[0.057,0.159]$} & 0.079 & {$[0.049,0.127]$} & 0.208 & {$[0.127,0.331]$} \\
\hline $\operatorname{In}\left(\sigma_{\zeta C}^{2}\right)$ & 1.359 & {$[0.946,2.005]$} & 1.158 & {$[0.801,1.737]$} & 1.791 & {$[1.243,2.675]$} \\
\hline $\operatorname{In}\left(\sigma_{\varepsilon C}^{2}\right)$ & 1.588 & {$[1.081,2.429]$} & 1.436 & {$[0.987,2.144]$} & 1.830 & {$[1.265,2.740]$} \\
\hline
\end{tabular}

Within-person standardization

Standardized parameter estimates

(averaged across clusters)

$\begin{array}{lrcrcrc}\varphi_{C} & 0.539 & {[0.479,0.600]} & 0.543 & {[0.477,0.615]} & 0.485 & {[0.414,0.553]} \\ \varphi_{D B} & 0.202 & {[0.111,0.292]} & 0.206 & {[0.113,0.296]} & 0.168 & {[0.064,0.260]} \\ \varphi_{D B D n C} & -0.121 & {[-0.195,-0.051]} & -0.165 & {[-0.242,-0.093]} & -0.142 & {[-0.237,-0.063]} \\ \varphi_{C o n D B} & -0.214 & {[-0.270,-0.154]} & -0.196 & {[-0.263,-0.125]} & -0.312 & {[-0.374,-0.241]} \\ \operatorname{In}\left(\sigma_{\zeta C}^{2}\right) & 0.506 & {[0.429,0.569]} & 0.505 & {[0.428,0.569]} & 0.428 & {[0.338,0.491]} \\ \operatorname{In}\left(\sigma_{\varepsilon C}^{2}\right) & 0.259 & {[0.191,0.340]} & 0.349 & {[0.285,0.425]} & 0.367 & {[0.313,0.438]}\end{array}$

Between-person standardization

$\left.\begin{array}{llllllll}\beta & -0.533 & {[-0.693,-0.331}\end{array}\right] \quad-0.445 \quad[-0.620,-0.239] \quad-0.511 \quad[-0.656,-0.327]$

Within-level (averaged across

clusters)

\begin{tabular}{lllllll}
$D B$ & .218 & {$[.171, .269]$} & .251 & {$[.206, .296]$} & .229 & {$[.188, .275]$} \\
$C$ & .494 & {$[.430, .571]$} & .495 & {$[.430, .571]$} & .572 & {$[.509, .662]$} \\
$Y$ & .741 & {$[.660, .809]$} & .651 & {$[.574, .715]$} & .633 & {$[.562, .687]$} \\
$\begin{array}{l}\text { Between-level } \\
D B\end{array}$ & .285 & {$[.109, .480]$} & .198 & {$[.057, .385]$} & .261 & {$[.107, .430]$} \\
\hline
\end{tabular}

Note: Within-person standardized parameters are within-person-level standardized estimates averaged over clusters. The estimated parameters denote posterior medians, and 95\% confidence intervals (CIs) denote Bayesian credibility intervals. The results for valence stem from Model 1 , the results for tense arousal stem from Model 2, and those for self-esteem stem from Model 3. Note that $R^{2}$ measures for dysfunctional behavior do not correct for measurement error (unexplained variance contains measurement error as well as innovation variances), those given for construct $C$ denote explained variance with respect to the measurement-error-free latent construct, and those for $Y$ refer to explained variance in the observed variables on the withinlevel. $\beta=$ regression coefficients of regressing $D B$ on the respective construct on the between-person/trait level; $C=$ construct included in the respective model, either tense arousal, valence, or self-esteem; $D B=$ dysfunctional behavior (dummy coded with 1 in case of dysfunctional behavior was shown); $E\left(\mu_{C}\right)=$ expectation; $\gamma_{\varphi D B o n C}=$ average slope (fixed effect) of the probit regression of $D B$ at time $t$ on the relevant predictor $C$ at time $t-1 ; \gamma_{\varphi D B}=$ average autoregressive effect (fixed effect) of $D B ; \gamma_{\varphi C}=$ average autoregressive effect (fixed effect) of the respective construct $C$; $\gamma_{\sigma \varepsilon C}=$ mean of the $\log$ measurement error variance of construct $C ; \gamma_{\zeta \zeta C}=$ mean of the log innovation variance of construct $C$; $\ln \left(\sigma_{\zeta C}^{2}\right)=\operatorname{logarithm}$ of the random, personspecific innovation variance; $\ln \left(\sigma_{\varepsilon C}^{2}\right)=$ logarithm of the random, person-specific measurement error variance; $\mu_{C}=$ person-specific latent mean (trait value) of the relevant construct $C$ across measurement occasions; $\varphi_{D B}=$ random, person-specific autoregressive effect of $D B$; $\varphi_{C}=$ random, personspecific autoregressive effect of the relevant construct; $\varphi_{D B o n C}=$ random, person-spec fic slope of the probit regression of $D B$ at time $t$ on the respective predictor $C$ at time $t-1 ; \varphi_{C o n D B}=$ random, person-specific slope of the regression of the respective construct $C$ at time $t$ on dysfunctional behavior in the preceding time period; $\tau=$ threshold of the dichotomous dependent variable $D B ; \theta=$ between-level residual variance of the latent response variable of $D B ; Y=$ Observed indicator variable for construct $C$; SEM $=$ structural equation modeling. 
did not have a significant effect on dysfunctional behavior on the temporal, within-person level. The combination of self-esteem and valence together with autoregressive effects explained around $25.7 \%$ of the within-person temporal deviations of a person's inclination to engage in dysfunctional behavior from this person's individual average level (3.9\% more than valence and $2.8 \%$ more than self-esteem as sole predictors besides autoregressive effects). On the between-person level (trait), only valence remained a significant predictor $\left(\beta_{V A}=-0.415,95 \%\right.$ confidence interval $[\mathrm{CI}]=$ $\left.[-0.724,-0.083] ; \beta_{S}=-0.189, \mathrm{CI}=[-0.487,0.131]\right)$. With respect to stable between-person differences, valence and self-esteem together explained around $34.0 \%$ of the variance in dysfunctional behavior (5.5\% more than valence alone; $7.9 \%$ than self-esteem alone).

Tense arousal and self-esteem. In the combination of tense arousal with self-esteem, momentary tense arousal had the comparatively larger effect compared with momentary self-esteem on the within-person level; higher calmness/less tension reduced the probability of engaging in dysfunctional behavior in the following hour $\left(\varphi_{\text {DBonTA }}=-0.124, \mathrm{CI}=[-0.229,-0.025]\right.$ and $\varphi_{\text {DBonS }}=-0.066$, $\mathrm{CI}=[-0.198,0.064])$. The combination of self-esteem and tense arousal together with autoregressive effects explained around $27.0 \%$ of the within-person, temporal variation (1.9\% more than tense arousal and $4.1 \%$ more than selfesteem as sole predictors besides autoregressive effects). On the between-person level (trait), only self-esteem remained a significant predictor of dysfunctional behavior $\left(\beta_{T A}=-0.250, \mathrm{CI}=[-0.528,0.045] ; \beta_{S}=-0.294, \mathrm{CI}=[-0.551\right.$, $-0.013])$. A combination of tense arousal and self-esteem explained $26.2 \%$ of the variance in dysfunctional behavior on the stable between-person level $(6.4 \%$ more than tense arousal alone; $0.1 \%$ more than self-esteem alone).

\section{Effects of dysfunctional behavior on subsequent affect and self-esteem (Hypotbesis 4)}

First, we examined Models 1 to 3, which used only a single parameter (valence, tense arousal, or self-esteem) and not the combination of two of them (see Table 1). Cross-lagged effects of dysfunctional behaviors on subsequent affect and self-esteem measures indicated that if a patient showed a higher probability compared with this patient's average inclination to engage in dysfunctional behavior in the time period preceding the outcome, subsequent measures of self-esteem and valence tended to decrease and tension tended to increase (within-person standardized coefficients $\varphi_{\text {ConDB }}$ of $-0.312,-0.214$, and -0.196 ; again, note that tense arousal is coded such that high values indicate low tension). This means that contrary to our hypothesis, tense arousal and valence (as well as self-esteem) worsened after instances of dysfunctional behaviors. Second, we examined Models 4 and 5, which combined parameters within single analyses. Regarding the effects of dysfunctional behavior on subsequent affect and self-esteem, an increase in the probability to engage in dysfunctional behavior had a significant negative effect (i.e., it significantly reduced valence, reduced tense arousal, and thus increased tension) and reduced selfesteem in both models.

Additional results: $R^{2}$, reliability, and innovation covariance. $R^{2}$ values for tense arousal, valence, and tense arousal in the single predictor models (Models 1-3) indicated that predictability was slightly higher for selfesteem; $57.2 \%$ of the variance in within-person, temporal deviations of self-esteem values from a person's habitual level were predictable by the autoregressive effect along with dysfunctional behavior, whereas for valence and tense arousal, $49.4 \%$ and $49.5 \%$ of the variance were predictable. Adding cross-lagged effects between affect and self-esteem beyond and above autoregressive effects as well as the effect of preceding within-level dysfunctional behavior (Models 4 and 5), up to $86.5 \%, 84.1 \%$, and $76.8 \%$ of the variance in within-person deviations across stable trait levels of self-esteem, valence, and tense arousal, respectively, were predictable over time. $R^{2}$ values of the observed variables $Y_{\text {TAit }}, Y_{\text {VAit }}$, and $Y_{\text {Sit }}$ provided values of average person-specific reliabilities, which indicated that reliability was high for all constructs (.732-.849).

As expected, all autoregressive effects as well as the cross-lagged effects of affect on self-esteem and selfesteem on affect were positive. We considered the correlations of the random innovations across constructs at the same time point, and unexplained parts of valence and self-esteem on the within-level correlated to approximately .62 $(S D=.25)$ and unexplained within-level parts of tense arousal correlated with unexplained within-level parts of self-esteem to approximately $.60(S D=.24) .{ }^{4}$ That is, there was a tendency that, at a given time point, the higher a patient's deviation of valence or tense arousal from the value expected on the basis of autoregressive and cross-lagged effects, the higher this person's deviation of self-esteem was from its expected value on that same time point (into the same direction). Hence, unobserved influences at a specific time point tended to affect not only tense arousal or valence but also self-esteem in a similar way.

\section{Additional results: latent correlations between random effects on the between-person level}

Inspection of the latent correlations between the random effects of Models 1 through 3 on the betweenperson level (see Table S2 in the Supplemental Material) 


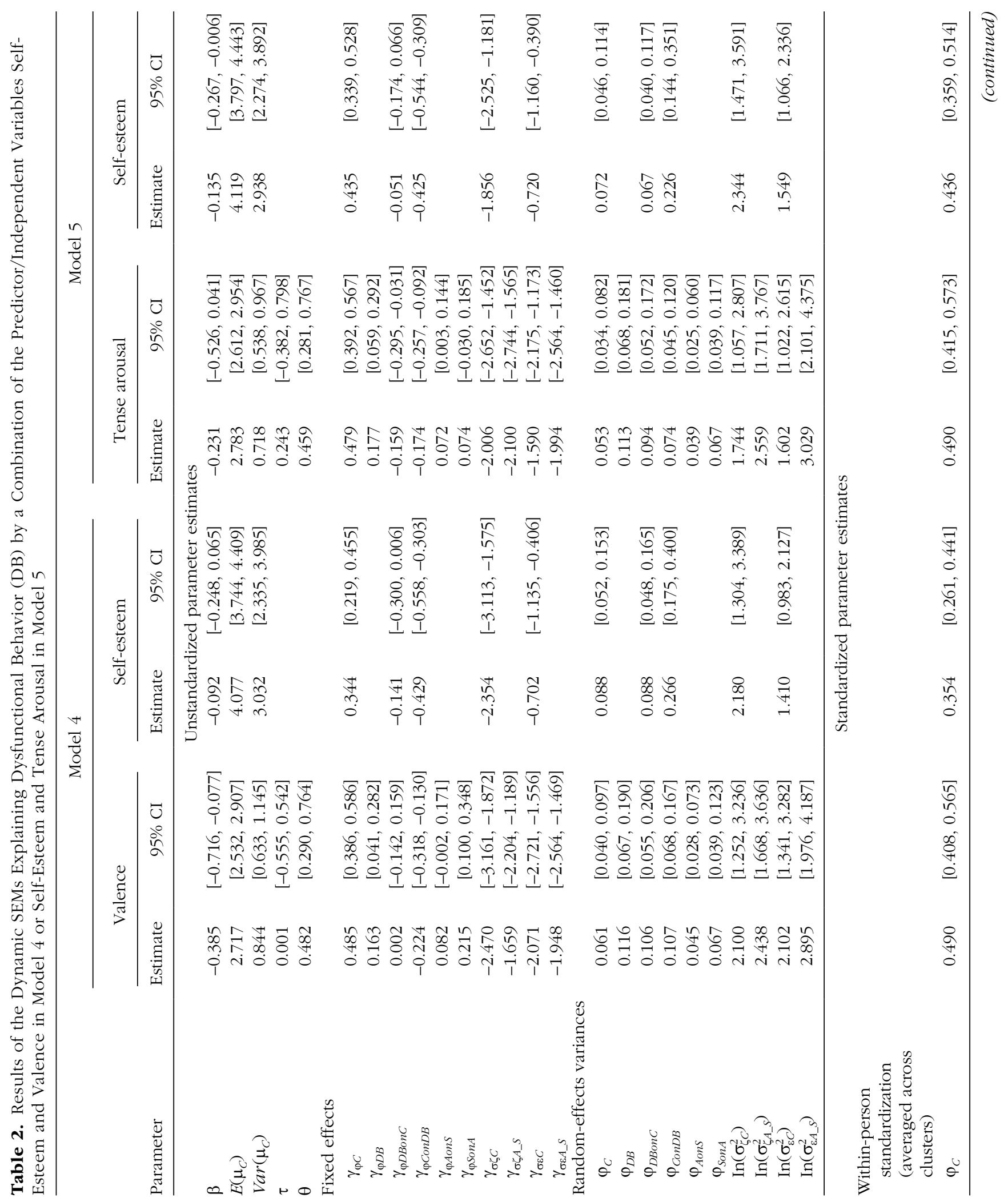




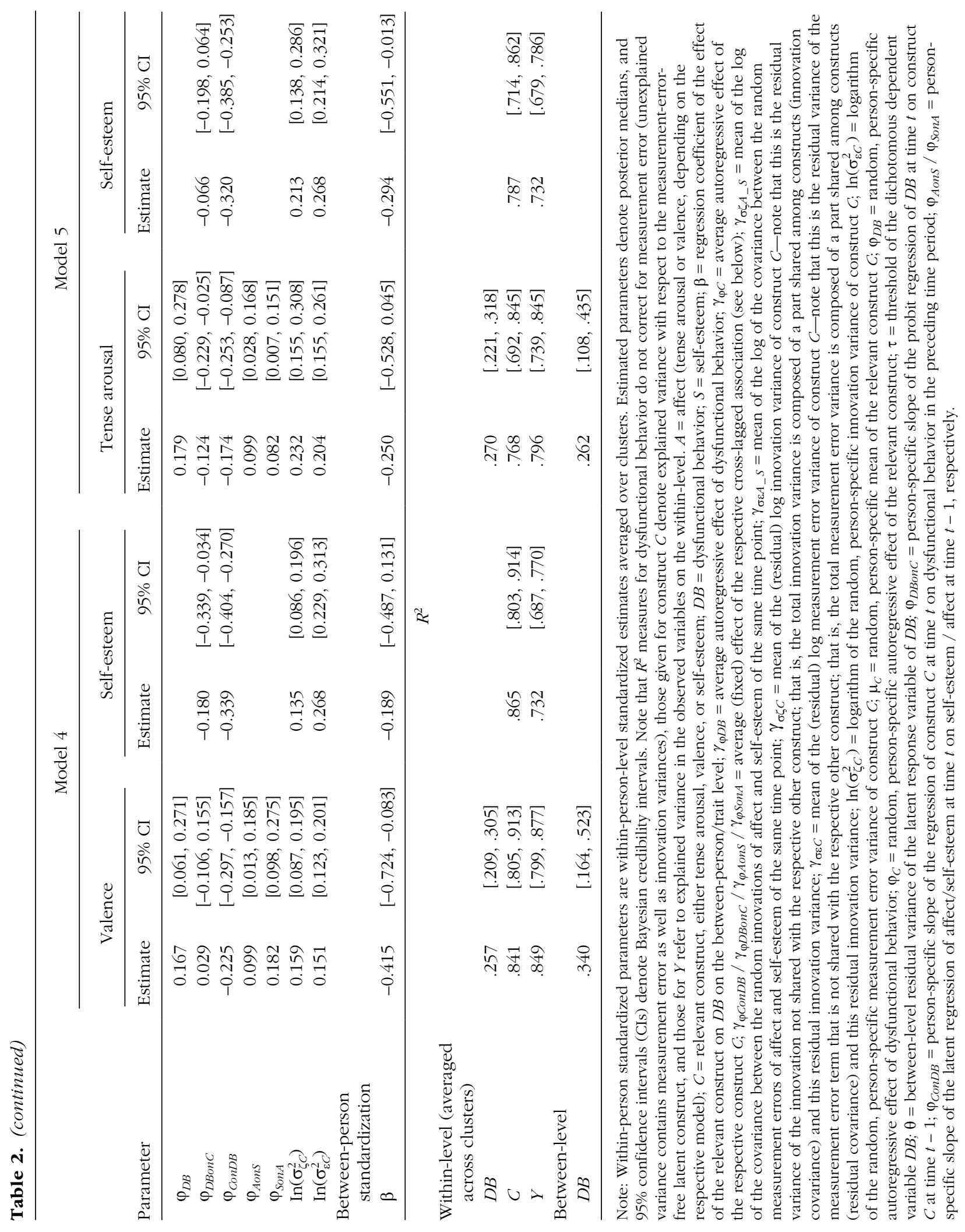


revealed the following patterns. The higher a person's trait level of valence or self-esteem, the higher the person's innovation variance was on the same construct$r\left(\mu_{C}, \ln \left(\sigma_{\zeta C}^{2}\right)\right)=.311$ and .460 , respectively. Stated differently, patients with low stable levels of self-esteem or valence tended to show less unexplained variability across time in their self-esteem and valence, respectively. That is, they tended to stay at their lower trait level across time with less variation.

With respect to self-esteem, higher autoregressive effects of self-esteem or dysfunctional behavior tended to go along with less reciprocal influences of dysfunctional behavior on self-esteem and self-esteem on dysfunctional behavior across time, respectively- $r\left(\varphi_{S}, \varphi_{\text {SonDB }}\right)=.319$ and $r\left(\varphi_{D B}, \varphi_{D B o n S}\right)=.374$ for negative fixed-effects values of $\gamma_{\varphi S O n D B}$ and $\gamma_{\varphi D B o n s}$ (i.e., $\varphi_{\text {SonDB }}$ and $\varphi_{D B o n S}$ tended to be closer to 0 for higher $\varphi_{S}$ and $\left.\varphi_{D B}\right)$. Similar association patterns for valence and tense arousal were nonsignificant.

Furthermore, a larger innovation variance of selfesteem was associated with a stronger effect of dysfunctional behavior on self-esteem-r $r\left(\varphi_{\text {SonDB }}, \ln \left(\sigma_{\zeta S}^{2}\right)\right)=$ -.457 . That is, for patients that have a larger amount of unexplained variability in self-esteem across time, an increase in the probability to engage in dysfunctional behavior in the temporally preceding time period had a stronger negative impact on self-esteem at the subsequent measurement occasion (i.e., self-esteem was reduced to larger amounts). Stated differently, for patients that show less variability in self-esteem, the effect of dysfunctional behavior on self-esteem was less prominent. This association was weaker and not significant for valence and tense arousal. Note that although not significant, for valence and tense arousal, a higher innovation variance seemed to be associated with a weaker effect of valence or tense arousal on reducing the probability of reporting dysfunctional behavior$r\left(\varphi_{\text {DBonTA }}, \ln \left(\sigma_{\zeta T A}^{2}\right)\right)=.464$ and $r\left(\varphi_{\text {DBonVA }}, \ln \left(\sigma_{\zeta V A}^{2}\right)\right)=.410$, n.s. (see Table S3 in the Supplemental Material).

The latent correlations between the random effects of Models 4 and 5 are presented in Table S3. Additional correlations in these models concerned associations between random effects of valence and tense arousal with those of self-esteem. Results showed that higher trait levels of tense arousal $\left(\mu_{T A}\right)$ or valence $\left(\mu_{V A}\right)$ were associated with higher trait levels of self-esteem $\left(\mu_{S E}\right.$; correlations $=.692$ and .774 , respectively). For a combination of valence and self-esteem, the larger a patient's cross-lagged effect of valence on dysfunctional behavior, the smaller this person's cross-lagged effect of self-esteem on dysfunctional behavior tended to be and vice versa $(r=-.541$; correlation not significant for the combination of tense arousal and self-esteem, $r=$ -.351). That is, it appeared that there is a tendency that either valence or self-esteem has a large or larger impact on the probability to show dysfunctional behavior for a specific person. Furthermore, the stronger self-esteem is affected by preceding dysfunctional behavior, the stronger valence is affected by preceding dysfunctional behavior across persons $(r=.712$; similar effect not found for tense arousal). For a combination of tense arousal and self-esteem, the cross-lagged effects of tense arousal on self-esteem and the crosslagged effect of self-esteem on tense arousal were positively correlated $(r=.535)$, which indicates that these two constructs are highly reciprocally interconnected for some persons (and less for others). For further correlations, see Table S3 in the Supplemental Material. Note that innovation and measurement error variances were not entered into the between-level covariance matrix in Models 4 and 5.

\section{Discussion}

In our study, we sought to test the core predictions of the benefits-and-barriers model (Hooley \& Franklin, 2018), which recently proposed that not only affect but also self-esteem is central to dysfunctional behavior. Indeed, and in accordance with our hypotheses, high momentary negative affect (i.e., negative valence and high tension) predicted upcoming dysfunctional behaviors. This finding was evident in Model 1 (with valence as single predictor), Model 2 (with tense arousal as single predictor), as well as Model 5 (combining tense arousal and self-esteem). This finding is in line with the benefits-and-barriers model as well as prior e-diary studies. Whereas the time intervals in prior studies ranged between 1.33 (Houben et al., 2017) and 2 to 3 hr (e.g., Andrewes et al., 2017a), we used 1-hr intervals (i.e., the highest sampling frequency to date). We propose that high sampling-frequency assessments are more effective when investigating short-term antecedents of dysfunctional behaviors than the less frequent samplings used in previous studies (Kockler et al., 2018). Moreover, our result fits both patients' retrospective reports of antecedents of dysfunctional behaviors (Kleindienst et al., 2008) as well as theoretical assumptions (Hooley \& Franklin, 2018; Linehan, 1993). For modeling short-term antecedents, high compliance is also necessary because both current and preceding ratings must be available. In this regard, our compliance rate $(84.86 \%)$ was advantageous compared with earlier studies with mostly considerably lower adherence rates (e.g., 52\%, Andrewes et al., 2017a; 65\%, Houben et al., 2017); only two studies reported compliance rates above 70\% (74\%, Scala et al., 2018; 90\%, Selby \& Joiner, 2013). In addition to negative affect, low momentary self-esteem also predicted upcoming dysfunctional 
behaviors (Model 3), which was not explicitly postulated as such in the benefits-and-barriers model. Nevertheless, momentary tense arousal had the strongest predictive effect on subsequent reports of dysfunctional behaviors on the within-person level.

Furthermore, we found that, consistent with the benefits-and-barriers model, the higher a person's trait level of self-esteem, the less likely it is that this person would show dysfunctional behavior in general (evident in Model 3, with self-esteem as single predictor, as well as Model 5, combining tense arousal and self-esteem). In addition, we found an association between a person's trait level of valence and tense arousal and engaging in dysfunctional behavior; trait self-esteem and valence had a higher impact on the general disposition to show dysfunctional behavior compared with tense arousal. Although the benefits-and-barriers model does not explicitly include momentary self-esteem or trait affect in having an influence on dysfunctional behaviors, it does not explicitly exclude them either. Therefore, the findings that low momentary self-esteem predicts dysfunctional behavior and that higher trait levels of negative affect predict engaging in dysfunctional behaviors do not contradict the benefits-andbarriers model, especially given that the hypothesized predictors most frequently showed the strongest effects. Because we gathered a comprehensive e-diary data set of very high data quality (i.e., with high-frequency sampling and high adherence rates) and, for the first time, used a fully cross-lagged multivariate latent variable model that accounted for measurement error and varying time intervals between measurements, we feel quite confident in our reported findings. Nonetheless, more empirical and theoretical studies differentiating between momentary and trait self-esteem are warranted to further examine their associations with dysfunctional behaviors. This is especially important given that numerous studies expanded the meaning of self-esteem by differentiating between the global level of selfesteem in general and self-esteem instability (i.e., the extent to which an individual experiences short-term fluctuations in self-esteem, e.g., Kernis, 2005). Selfesteem instability has been associated with psychological adjustment and functioning in healthy subjects' everyday lives (for an overview, see Santangelo et al., 2017).

Contrary to our hypothesis regarding the consequences of dysfunctional behaviors, these behaviors led to more negative valence and increases in tension (as well as a decrease in momentary self-esteem). This finding conflicts with the assumptions of the benefitsand-barriers model and challenges common beliefs about the affect-regulatory effect of dysfunctional behaviors. However, this is in line with findings from previous e-diary studies, especially those with a high sampling frequency, that showed a deteriorating negative affect after engaging in dysfunctional behaviors (Houben et al., 2017; Snir et al., 2015). Compared with previous studies, the high sampling frequency in our study resulted in smaller time intervals between consecutive ratings and thus between occurrences of dysfunctional behaviors and subsequent ratings of the current state. Moreover, we analyzed a comprehensive data set of 119 subjects with a very good adherence rate and yielded higher numbers of dysfunctional behaviors than previous studies. In addition, we used, for the first time, a fully cross-lagged multivariate latent variable model that accounted for measurement error and varying time intervals between measurements, which eliminated problematic issues of earlier studies. Nonetheless, we did not find the hypothesized improvement of affective state after reports of dysfunctional behaviors. Thus, our study illustrates that the affectregulating effect of dysfunctional behaviors is at best short-lived $(<1 \mathrm{hr})$ and that these behaviors actually increase negative valence and aversive tension in a time frame as short as $1 \mathrm{hr}$. From a clinical perspective, a possible explanation might be that dysfunctional behaviors have an affect-regulatory effect in the early stages of the development of symptoms. However, this effect might wear off with increasing duration of the disorder so that over the long term, dysfunctional behavior might change from a maintaining factor to a conditioned habit that is consolidated in the brain. More studies are necessary to support this hypothesis.

To summarize, using the latest methodology for data assessment and statistical analyses in combination with a comprehensive data set, we revealed that tense arousal was the strongest predictor for reports of dysfunctional behaviors on the within-person level and that self-esteem and valence were the strongest predictors on the between-person level. Moreover, combining affective state and self-esteem as a within- and between-person predictor substantially increased the amount of explained variance when predicting occurrences of dysfunctional behaviors. Contrary to our expectations, affective state did not improve but worsened after reports of dysfunctional behaviors (as did self-esteem).

Clinical implications. Elucidating the factors associated with dysfunctional behaviors among patients with BPD has great clinical and public-health significance. The management of aversive negative affective states is particularly important in reducing dysfunctional behaviors in patients with BPD. This highlights the importance of treatment approaches that address emotion dysregulation and teach skills on how to cope with those states, such as dialectical behavior therapy (Linehan, 1993). However, 
given the finding that high self-esteem constitutes a barrier and a protective factor against dysfunctional behaviors, it is likely that building or reestablishing self-esteem reduces dysfunctional behaviors among patients with BPD. Therefore, reestablishing a positive association with the self may be a particularly important treatment target to reduce dysfunctional behaviors in BPD. Of course, our investigation cannot explain which treatment strategy is more successful. However, treatment studies with separate modules focusing on either emotion regulation or building a positive self may be particularly enlightening. Furthermore, our finding that dysfunctional behaviors lead to more negative valence and increases in tension on the next assessment challenges common beliefs about the affect-regulatory effect of dysfunctional behaviors and should inform practice in clinical care (i.e., psychoeducation). Patients should be educated that engaging in dysfunctional behaviors is associated with increased negative valence and higher tension (as well as a decrease in self-esteem). Therefore, the relieving affect-regulatory effect of these behaviors is short-lived at best $(<1 \mathrm{hr})$, and engaging in them leads to worsening of affective state and self-esteem. This in turn increases the probability of engaging in dysfunctional behaviors.

Limitations. Several limitations of our study must be acknowledged. We broadened the scope of the benefitsand-barriers model and tested its assumptions examining dysfunctional behaviors in general (and not only nonsuicidal self-injury). This was done by combining the most frequently reported dysfunctional self-harming behaviors in BPD into a single dysfunctional behaviors variable in our statistical model. This can be controversially discussed. It is possible that all these behaviors serve the same purpose of emotion regulation, constituting dysfunctional regulation strategies, and are therefore ideally considered together. However, it could also be argued that direct (i.e., nonsuicidal self-injuries) and more indirect forms of self-injury, such as abusing substances or binge eating, might have different functions (Hooley \& Franklin, 2018). Previous e-diary studies showed that capturing instances of nonsuicidal self-injury is challenging because of its rare occurrence. Those studies dealt with the low occurrences by aggregating data on the day level (e.g., Ammerman, Olino, Coccaro, \& McCloskey, 2017), using low sampling frequencies (e.g., Snir et al., 2015), and investigating urges instead of acts (e.g., Scala et al., 2018), probably contributing to the heterogeneous findings. An additional concern is that low rates of acts of nonsuicidal self-injury also reduce the number of participants for the statistical analysis. In our comprehensive sample of 119 patients, only 38 (i.e., 31.93\%) reported at least one act of nonsuicidal self-injury, and nonsuicidal self-injuries constituted only $8.72 \%$ of the total number of dysfunctional behaviors reported (see Table S1 in the Supplemental Material). Thus, limiting the analyses to only nonsuicidal self-injuries would have substantially reduced the number of participants and the instances of dysfunctional behaviors available for the statistical analyses. Future studies with larger samples may address potential differences between various forms of dysfunctional behaviors. However, compared with the existing literature, we have substantially more reports of dysfunctional behaviors that could be included in the analyses.

Second, given that only female participants were included in our study, the generalizability of the findings is limited, and the results may not be valid for male BPD patients. However, the use of an entirely female sample also reduced the heterogeneity of the sample, which may have been useful given the literature on sex differences in affect (Fujita, Diener, \& Sandvik, 1991) and self-esteem (Kling, Hyde, Showers, \& Buswell, 1999). Moreover, BPD patients were diagnosed with a variety of comorbid Axis I and Axis II disorders, and no control group was included. No statement can be made regarding whether our findings are independent of any comorbidity or whether they are BPD-specific. However, in BPD, comorbidity is the rule rather than the exception (Sanislow, Marcus, \& Reagan, 2012). Regarding the specificity of our findings for BPD, we focused on patients with this disorder to increase the chances of gathering significant numbers of dysfunctional behaviors. At the same time, we assume that the processes involved in dysfunctional behaviors are not specific to patients with BPD but constitute transdiagnostic phenomena (as does the benefits-and-barriers model assume). Still, future studies have to show the transdiagnostic nature of these processes empirically.

Third, we used state-of-the-art statistics (i.e., dynamic SEM) to test the assumptions of the benefits-and-barriers model. Because the models of the dynamic SEM framework employed in our study are quite new, researchers know little with respect to their performance under different sample sizes or assessment points. In a small simulation study that investigated a reduced variant of the employed model, Schuurman and Hamaker (2019) found parameter estimates to be acceptable for a sample size of 100 persons with 100 measurement time points, although estimates of the variances and covariances of random regression coefficients should be interpreted with caution. Detailed simulations for a comparable model extended by a categorical variable are still missing. However, Asparouhov and Muthén (2019) found the estimate of the average autocorrelation to be unbiased in an analogous univariate categorical variable model with as few as 20 observations per cluster. In contrast to previously applied modeling techniques, our model not only incorporates interindividual differences 
in lagged relations and unexplained variability (innovation variance) but also corrects for potential measurement error and unevenly spaced observations, which might otherwise distort estimates of autoregressive and lagged effects.

Fourth, we conceptualized a person's trait as a variable's stable component (measurement-error-free) across the assessment period. These traits might differ from those obtained by traditional single-time-point questionnaire assessments. However, this procedure to measure traits increased measurement accuracy and avoided cognitive biases (e.g., recall biases) in global judgments of global aspects (Stone \& Litcher-Kelly, 2006). In addition, a longer total assessment period might be helpful to better separate traits from momentary states, but this would conflict with the idea of having an even higher sampling frequency to better picture short-term consequences of dysfunctional behavior.

Despite these limitations, our study significantly deepens the understanding of the psychological processes involved in dysfunctional behaviors in BPD. Because we extended prior e-diary research on dysfunctional behaviors in BPD, our study conducted in patients' everyday lives builds on growing evidence for the protective effect of a positive view of oneself, as is suggested in the recent benefits-and-barriers model (Hooley \& Franklin, 2018). Our findings demonstrate that self-esteem plays an important role in occurrences of dysfunctional behaviors because high self-esteem acts as a buffer against dysfunctional behaviors in individuals with BPD, which lowers the tendency to engage in dysfunctional behaviors in general. In addition, in accordance with the model and prior e-diary studies, we found that negative affective states directly predict reports of dysfunctional behaviors in daily life. Our findings also indicate that low momentary self-esteem predicts occurrences of dysfunctional behaviors and that trait valence and tense arousal also have an effect on engaging in dysfunctional behaviors. Our study illustrates that the affect-regulating function of dysfunctional behaviors is short-lived at best and that those behaviors may actually increase negative affect. Thus, our findings, which are based on a large e-diary study using a high sampling frequency (i.e., examining the antecedents and consequences of dysfunctional behaviors on a shorter time scale than previous studies), are partly in accordance with the predictions stated by the benefits-and-barriers model (Hooley \& Franklin, 2018): Self-esteem constitutes an important factor in dysfunctional behaviors because a high level of trait self-esteem prevents patients from engaging in self-harming behavior, whereas acts of self-harming behavior are best predicted by states of high negative affect on the within-person level, especially states of high tension.
Contrary to the benefits-and-barriers model, we found no affect-regulating function of dysfunctional behaviors but rather a worsening of affective state after reports of dysfunctional behaviors. This finding challenges common beliefs about affect regulation in dysfunctional behaviors and should inform practice in clinical care (i.e., psychoeducation).

\section{Transparency}

Action Editor: Scott O. Lilienfeld

Editor: Scott O. Lilienfeld

Author Contributions

P. S. Santangelo and J. Holtmann are equally contributing first authors. U. W. Ebner-Priemer and M. Eid developed the study concept and obtained funding for the study. P. S. Santangelo, T. D. Kockler, and S. Koudela-Hamila performed the data collection. Data collection was overseen by U. W. Ebner-Priemer and M. Bohus. All of the authors contributed to the study design and analytic plan. J. Holtmann and G. Hosoya performed the data analysis and interpretation in collaboration with M. Eid. P. S. Santangelo, J. Holtmann, and U. W. Ebner-Priemer drafted the manuscript, and all of the authors provided critical revisions. All of the authors approved the final manuscript for submission.

Declaration of Conflicting Interests

The author(s) declared that there were no conflicts of interest with respect to the authorship or the publication of this article.

Funding

This work was supported by Deutsche Forschungsgemeinschaft Grants EB 364/6-1 and EI 379/10-1.

\section{Notes}

1. Note that measurement error terms may also capture within-person fluctuations that are specific to one measurement time point and do not carry over to later time points (Schuurman \& Hamaker, 2019). The logarithms of the person-specific residual variances and covariances were modeled to be (univariate) normally distributed with a mean across persons and a variance of person-specific 
deviations on the between-level. Thereby, reliabilities were modeled to be person-specific. For details on the estimation of personspecific reliabilities, see Schuurman and Hamaker (2019).

2 . The logarithms of the innovation variances were assumed to be normally distributed and modeled with a mean and a variance on the between-person level (i.e., the random variances were assumed to be log-normally distributed on the between-person level; Hamaker et al., 2018). Note that in the models combining dysfunctional behavior with either affect or self-esteem (Models 1-3), the log innovation variances are entered in a multivariate normal distribution along with all other between-level variables (including random effects) on the between-level. For reasons of model parsimony, log innovation variances and covariances were modeled with univariate normal distributions in the case of models combining dysfunctional behavior with affect and self-esteem. For further details, see Model Definitions in the Supplemental Material.

3. This modeling strategy was chosen in case of the dichotomous variable dysfunctional behavior only, given that dynamic SEMs for latent variables measured by $Y_{i t}^{*}$ for categorical variables with few categories might need substantial sample sizes of more than a thousand measurement time points (Asparouhov et al., 2018). The chosen modeling strategy for dichotomous observed variables was proposed in Asparouhov and Muthén (2019) and works well for sample sizes as small as 100 clusters with 20 time points for a model considering one dichotomous variable (Asparouhov \& Muthén, 2019).

4. Correlations are averages across person-specific correlations calculated on the basis of estimated person-specific log-variances and log-covariances.

\section{References}

American Psychiatric Association. (1994). Diagnostic and statistical manual of mental disorders (4th ed.). Washington, DC: Author.

American Psychiatric Association. (2013). Diagnostic and statistical manual of mental disorders (5th ed.). Washington, DC: Author.

Ammerman, B. A., Olino, T. M., Coccaro, E. F., \& McCloskey, M. S. (2017). Predicting nonsuicidal self-injury in borderline personality disorder using ecological momentary assessment. Journal of Personality Disorders, 31, 844-855. doi:10.1521/pedi_2017_31_278

Andrewes, H. E., Hulbert, C., Cotton, S. M., Betts, J., \& Chanen, A. M. (2017a). An ecological momentary assessment investigation of complex and conflicting emotions in youth with borderline personality disorder. Psychiatry Research, 252, 102-110. doi:10.1016/j.psychres.2017.01.100

Andrewes, H. E., Hulbert, C., Cotton, S. M., Betts, J., \& Chanen, A. M. (2017b). Ecological momentary assessment of nonsuicidal self-injury in youth with borderline personality disorder. Journal of Personality Disorders, 8 , 357-365. doi:10.1037/per0000205

Asparouhov, T., Hamaker, E. L., \& Muthén, B. (2018). Dynamic structural equation models. Structural Equation Modeling, 25, 359-388. doi:10.1080/10705511.2017.1406803

Asparouhov, T., \& Muthén, B. (2010). Bayesian analysis using Mplus: Technical implementation: Version 3. Retrieved from http://statmodel.com/download/Bayes3.pdf
Asparouhov, T., \& Muthén, B. (2019). Latent variable centering of predictors and mediators in multilevel and time-series models. Structural Equation Modeling: A Multidisciplinary Journal, 26, 119-142. doi:10.1080/10705511.2018.1511375 Borton, J. L., Crimmins, A. E., Ashby, R. S., \& Ruddiman, J. F. (2012). How do individuals with fragile high self-esteem cope with intrusive thoughts following ego threat? Self and Identity, 11, 16-35. doi:10.1080/15298868.2010.500935

Chapman, A. L., Gratz, K. L., \& Brown, M. Z. (2006). Solving the puzzle of deliberate self-harm: The experiential avoidance model. Behaviour Research and Therapy, 44, 371-394. doi:10.1016/j.brat.2005.03.005

Ebner-Priemer, U. W., Houben, M., Santangelo, P. S., Kleindienst, N., Tuerlinckx, F., Oravecz, Z., . . . Kuppens, P. (2015). Unraveling affective dysregulation in borderline personality disorder: A theoretical model and empirical evidence. Journal of Abnormal Psychology, 124, 186-198. doi:10 .1037/abn0000021

Ebner-Priemer, U. W., \& Sawitzki, G. (2007). Ambulatory assessment of affective instability in borderline personality disorder: The effect of the sampling frequency. European Journal of Psychological Assessment, 23, 238-247.

Fujita, F., Diener, E., \& Sandvik, E. (1991). Gender differences in negative affect and well-being: The case for emotional intensity. Journal of Personality and Social Psychology, 61, 427-434. doi:10.1037/0022-3514.61.3.427

Fydrich, T., Renneberg, B., Schmitz, B., Wittchen, H.-U., First, M. B., \& Benjamin, L. (1997). SKID-II: Strukturiertes klinisches Interview für DMS-IV. Achse II: Persönlichkeitsstörungen [Structured clinical interview for DSM-IV. Axis II: Personality disorders]. Göttingen, Germany: Hogrefe.

Guan, K., Fox, K. R., \& Prinstein, M. J. (2012). Nonsuicidal selfinjury as a time-invariant predictor of adolescent suicide ideation and attempts in a diverse community sample. Journal of Consulting and Clinical Psychology, 80, 842849. doi:10.1037/a0029429

Hamaker, E. L., Asparouhov, T., Brose, A., Schmiedek, F., \& Muthén, B. (2018). At the frontiers of modeling intensive longitudinal data: Dynamic structural equation models for the affective measurements from the COGITO Study. Multivariate Behavioral Research, 53, 820-841. doi:10.1080/ 00273171.2018 .1446819

Hasking, P., Whitlock, J., Voon, D., \& Rose, A. (2017). A cognitive-emotional model of NSSI: Using emotion regulation and cognitive processes to explain why people selfinjure. Cognition E Emotion, 31, 1543-1556. doi:10.1080/ 02699931.2016 .1241219

Hooley, J. M., \& Franklin, J. C. (2018). Why do people hurt themselves?: A new conceptual model of nonsuicidal selfinjury. Clinical Psychological Science, 6, 428-451. doi:10 $.1177 / 2167702617745641$

Houben, M., Claes, L., Vansteelandt, K., Berens, A., Sleuwaegen, E., \& Kuppens, P. (2017). The emotion regulation function of nonsuicidal self-injury: A momentary assessment study in inpatients with borderline personality disorder features. Journal of Abnormal Psychology, 126, 89-95. doi:10.1037/abn0000229

Jongerling, J., Laurenceau, J.-P., \& Hamaker, E. L. (2015). A multilevel AR(1) model: Allowing for inter-individual differences in trait-scores, inertia, and innovation variance. 
Multivariate Behavioral Research, 50, 334-349. doi:10.1080/ 00273171.2014 .1003772

Kernis, M. H. (2005). Measuring self-esteem in context: The importance of stability of self-esteem in psychological functioning. Journal of Personality, 73, 1569-1605. doi:10 .1111/j.1467-6494.2005.00359.x

Kleindienst, N., Bohus, M., Ludäscher, P., Limberger, M. F., Kuenkele, K., Ebner-Priemer, U. W., ... Schmahl, C. (2008). Motives for nonsuicidal self-injury among women with borderline personality disorder. Journal of Nervous and Mental Disease, 196, 230-236. doi:10.1097/ NMD.0b013e3181663026

Kling, K. C., Hyde, J. S., Showers, C. J., \& Buswell, B. N. (1999). Gender differences in self-esteem: A meta-analysis. Psychological Bulletin, 125, 470-500. doi:10.1037/00332909.125.4.470

Kockler, T. D., Santangelo, P. S., \& Ebner-Priemer, U. W. (2018). Investigating binge eating using ecological momentary assessment: The importance of an appropriate sampling frequency. Nutrients, 10(1). doi:10.3390/nu10010105

Linehan, M. (1993). Cognitive-behavioral treatment of borderline personality disorder. Diagnosis and treatment of mental disorders. New York, NY: Guilford Press.

Lobbestael, J., Leurgans, M., \& Arntz, A. (2011). Inter-rater reliability of the structured clinical interview for DSM-IV axis I disorders (SCID I) and axis II disorders (SCID II). Clinical Psychology \& Psychotherapy, 18, 75-79.

Marino, M. F., \& Zanarini, M. C. (2001). Relationship between EDNOS and its subtypes and borderline personality disorder. International Journal of Eating Disorders, 29, 349-353.

McGlashan, T. H., Grilo, C. M., Skodol, A. E., Gunderson, J. G., Shea, M. T., Morey, L. C., . . Stout, R. L. (2000). The Collaborative Longitudinal Personality Disorders Study: Baseline Axis I/II and II/II diagnostic co-occurrence. Acta Psychiatrica Scandinavica, 102, 256-264. doi:10.1034/ j.1600-0447.2000.102004256.x

McKenzie, K. C., \& Gross, J. J. (2014). Nonsuicidal self-injury: An emotion regulation perspective. Psychopathology, 47, 207-219. doi:10.1159/000358097

Muthén, L. K., \& Muthén, B. O. (2017). Mplus user's guide (8th ed.). Los Angeles, CA: Author.

Nock, M. K., \& Favazza, A. R. (2009). Nonsuicidal selfinjury: Definition and classification. In M. Nock (Ed.), Understanding nonsuicidal self-injury: Origins, assessment, and treatment (pp. 9-18). Washington, DC: American Psychological Association. doi:10.1037/11875-001

Nock, M. K., \& Prinstein, M. J. (2004). A functional approach to the assessment of self-mutilative behavior. Journal of Consulting and Clinical Psychology, 72, 885-890. doi:10 1037/0022-006X.72.5.885

Rodríguez-Blanco, L., Carballo, J. J., \& Baca-García, E. (2018). Use of ecological momentary assessment in non-suicidal self-injury: A systematic review. Psychiatry Research, 263, 212-219. doi:10.1016/j.psychres.2018.02.051

Rosenberg, M. (1965). Society and the adolescent self-image. Princeton, NJ: University Press.

Sanislow, C. A., Marcus, K. L., \& Reagan, E. M. (2012). Long-term outcomes in borderline psychopathology: Old assumptions, current findings, and new directions. Current Psychiatry Reports, 14, 54-61. doi:10.1007/s11920-011-0250-y
Santangelo, P. S., Bohus, M., \& Ebner-Priemer, U. W. (2014). Ecological momentary assessment in borderline personality disorder: A review of recent findings and methodological challenges. Journal of Personality Disorders, 28, 555-576. doi:10.1521/pedi_2012_26_067

Santangelo, P. S., Koenig, J., Kockler, T. D., Eid, M., Holtmann, J., Koudela-Hamila, S., . . Ebner-Priemer, U. W. (2018). Affective instability across the lifespan in borderline personality disorder: A cross-sectional e-diary study. Acta Psychiatrica Scandinavica, 138, 409-419. doi:10.1111/ acps. 12950

Santangelo, P. S., Reinhard, I., Koudela-Hamila, S., Bohus, M., Holtmann, J., Eid, M., \& Ebner-Priemer, U. W. (2017). The temporal interplay of self-esteem instability and affective instability in borderline personality disorder patients' everyday lives. Journal of Abnormal Psychology, 126, 1057-1065. doi:10.1037/abn0000288

Santangelo, P. S., Reinhard, I., Mussgay, L., Steil, R., Sawitzki, G., Klein, C., . . . Ebner-Priemer, U. W. (2014). Specificity of affective instability in patients with borderline personality disorder compared to posttraumatic stress disorder, bulimia nervosa, and healthy controls. Journal of Abnormal Psychology, 123, 258-272. doi:10.1037/a0035619

Scala, J. W., Levy, K. N., Johnson, B. N., Kivity, Y., Ellison, W. D., Pincus, A. L., . . Newman, M. G. (2018). The role of negative affect and self-concept clarity in predicting self-injurious urges in borderline personality disorder using ecological momentary assessment. Journal of Personality Disorders, 32(Suppl.), 36-57. doi:10.1521/pedi.2018.32.supp.36

Schuurman, N. K., Ferrer, E., Boer-Sonnenschein, M. de, \& Hamaker, E. L. (2016). How to compare cross-lagged associations in a multilevel autoregressive model. Psychological Methods, 21, 206-221. doi:10.1037/met0000062

Schuurman, N. K., \& Hamaker, E. L. (2019). Measurement error and person-specific reliability in multilevel autoregressive modeling. Psychological Methods, 24, 70-91. doi: $10.1037 /$ met0000188

Selby, E. A., Doyle, P., Crosby, R. D., Wonderlich, S. A., Engel, S. G., Mitchell, J. D., \& Le Grange, D. (2012). Momentary emotion surrounding bulimic behaviors in women with bulimia nervosa and borderline personality disorder. Journal of Psychiatric Research, 46, 1492-1500. doi:10.1016/j.jpsychires.2012.08.014

Selby, E. A., \& Joiner, T. E. (2013). Emotional cascades as prospective predictors of dysregulated behaviors in borderline personality disorder. Journal of Personality Disorders, 4, 168-174. doi:10.1037/a0029933

Snir, A., Rafaeli, E., Gadassi, R., Berenson, K., \& Downey, G. (2015). Explicit and inferred motives for nonsuicidal selfinjurious acts and urges in borderline and avoidant personality disorders. Journal of Personality Disorders, 6 , 267-277. doi:10.1037/per0000104

Solhan, M. B., Trull, T. J., Jahng, S., \& Wood, P. K. (2009). Clinical assessment of affective instability: Comparing EMA indices, questionnaire reports, and retrospective recall. Psychological Assessment, 21, 425-436. doi:10.1037/ a0016869

Stone, A. A., \& Litcher-Kelly, L. (2006). Momentary capture of real-world data. In M. Eid \& E. Diener (Eds.), Handbook of multimethod measurement in psychology (pp. 61-72). 
Washington, DC: American Psychological Association. doi: $10.1037 / 11383-005$

Trull, T. J. (2018). Ambulatory assessment of borderline personality disorder. Psychopathology, 51, 137-140. doi:10 $.1159 / 000486604$

Trull, T. J., \& Ebner-Priemer, U. W. (2013). Ambulatory assessment. Annual Review of Clinical Psychology, 9, 151-176. doi:10.1146/annurev-clinpsy-050212-185510

Wedig, M. M., Silverman, M. H., Frankenburg, F. R., Reich, D. B., Fitzmaurice, G., \& Zanarini, M. C. (2012). Predictors of suicide attempts in patients with borderline personality disorder over 16 years of prospective follow-up. Psychological Medicine, 42, 2395-2404. doi:10.1017/S0033291712000517

Whitlock, J., Muehlenkamp, J., Eckenrode, J., Purington, A., Baral Abrams, G., Barreira, P., \& Kress, V. (2013). Nonsuicidal self-injury as a gateway to suicide in young adults. The Journal of Adolescent Health, 52, 486-492. doi:10.1016/j.jadohealth.2012.09.010
Wilhelm, P., \& Schoebi, D. (2007). Assessing mood in daily life: Structural validity, sensitivity to change, and reliability of a short-scale to measure three basic dimensions of mood. European Journal of Psychological Assessment, 23, 258-267.

Wittchen, H. U., Wunderlich, U., \& Gruschwitz, S. (1997). SKID: Strukturiertes Klinisches Interview für DSM-IV Achse I: SCID: Structured clinical interview for DSM-IV Axis I. Göttingen, Germany: Hogrefe.

World Health Organization. (1992). ICD-10: International Statistical Classification of Diseases and Related Health Problems: 10th revision. Geneva, Switzerland: World Health Organization.

Zanarini, M. C., Laudate, C. S., Frankenburg, F. R., Wedig, M. M., \& Fitzmaurice, G. (2013). Reasons for self-mutilation reported by borderline patients over 16 years of prospective follow-up. Journal of Personality Disorders, 27, 783-794. doi:10.1521/pedi_2013_27_115 


\section{SKIT}

\section{Repository KITopen}

Dies ist ein Postprint/begutachtetes Manuskript.

Empfohlene Zitierung:

Santangelo, P. S.; Holtmann, J.; Hosoya, G.; Bohus, M.; Kockler, T. D.; Koudela-Hamila, S.; Eid, M.; Ebner-Priemer, U. W.

Within- and Between-Persons Effects of Self-Esteem and Affective State as Antecedents and Consequences of Dysfunctional Behaviors in the Everyday Lives of Patients With Borderline Personality Disorder.

2020. Clinical psychological science, 8

doi:10.5445/IR/1000119760

Zitierung der Originalveröffentlichung:

Santangelo, P. S.; Holtmann, J.; Hosoya, G.; Bohus, M.; Kockler, T. D.; Koudela-Hamila, S.; Eid, M.; Ebner-Priemer, U. W.

Within- and Between-Persons Effects of Self-Esteem and Affective State as Antecedents and Consequences of Dysfunctional Behaviors in the Everyday Lives of Patients With Borderline Personality Disorder.

2020. Clinical psychological science, 8 (3), 428-449.

doi:10.1177/2167702620901724 